\title{
STABILIZATION BY SPARSE CONTROLS FOR A CLASS OF SEMILINEAR PARABOLIC EQUATIONS*
}

\author{
EDUARDO $\mathrm{CASAS}^{\dagger}$ AND KARL KUNISCH ${ }^{\ddagger}$
}

\begin{abstract}
Stabilization problems for parabolic equations with polynomial nonlinearities are investigated in the context of an optimal control formulation with a sparsity enhancing cost functional. This formulation allows that the optimal control completely shuts down once the trajectory is sufficiently close to a stable steady state. Such a property is not present for commonly chosen control mechanisms. To establish these results it is necessary to develop a function space framework for a class of optimal control problems posed on infinite time horizons, which is otherwise not available.
\end{abstract}

Key words. semilinear parabolic equations, stabilization, optimal control, sparse controls

AMS subject classifications. 35K58, 49J20, 49J52, 49K20

DOI. $10.1137 / 16 \mathrm{M} 1084298$

1. Introduction. In recent years there has been significant interest in the topic of sparse optimal controls. These controls contribute towards the control objective and simultaneously shut down to zero as much as possible. Up to now optimal control problems with sparsity constraints have typically been investigated for tracking problems on finite time horizons. Sparsity with more general cost functionals was investigated in [6]. In this case the objective is to steer the trajectory of the controlled dynamical system to the desired state while simultaneously minimizing the support of the control. By an adapted choice of the cost functional, the sparsity structure can be influenced. The focus in previous work was set on controlling the sparsity structure in spatial directions. This led to choose controls in the spaces like $L^{2}\left(0, T ; L^{1}(\Omega)\right)$ or $L^{1}\left(\Omega ; L^{2}(0, T)\right)$, where, for technical reasons, the $L^{1}$-spaces have to be replaced by spaces of measures [5], [10], [7], unless other precautions as, for instance, constraints on the controls are taken [6], [9].

In the present paper we focus on optimal controls which exhibit temporal sparsity. This can be achieved by choosing a cost functional for the control variable which is nonsmooth in time. We shall concentrate on optimal control formulations for stabilization problems. It will be demonstrated that these problems are particularity well-suited to benefit from the sparsity structure. Specifically, if $y_{e}$ is a stable equilibrium of a dynamical system, optimal control strategy typically provides controls which asymptotically steer the system to $y_{e}$ with the control not shutting down to zero even if the controlled trajectory is already in the close vicinity of $y_{e}$. Such strategies can be based, for instance, on applying Riccati or Lyapunov techniques to the linearized system or on feedback mechanisms which respect the nature of the

\footnotetext{
*Received by the editors July 12, 2016; accepted for publication (in revised form) December 19, 2016; published electronically February 22, 2017.

http://www.siam.org/journals/sicon/55-1/M108429.html

Funding: The first author was supported by Spanish Ministerio de Economía y Competitividad under project MTM2014-57531-P. The second author was supported by the Austrian Science Fund (FWF) under grant SFB F32 (SFB "Mathematical Optimization and Applications in Biomedical Sciences") and by the ERC advanced grant 668998 (OCLOC) under the EU's H2020 research program.

${ }_{\dagger}^{\dagger}$ Departamento de Matemática Aplicada y Ciencias de la Computación, E.T.S.I. Industriales y de Telecomunicación, Universidad de Cantabria, 39005 Santander, Spain (eduardo.casas@unican.es).

${ }^{\ddagger}$ Institute for Mathematics and Scientific Computing, University of Graz, Heinrichstrasse 36, A8010 Graz, Austria (karl.kunisch@uni-graz.at).
} 
differential equation and the control objective, as, for example, feeding back some weighted difference between the state and $y_{e}$; see, for instance, [1], [2], [16]. With temporally sparse controls, on the other hand, it can be guaranteed that the optimal control will automatically shut down to zero in the vicinity of $y_{e}$. Of course, such a property cannot be expected unless $y_{e}$ is stable. In the present paper we develop the necessary concepts for a class of semilinear parabolic equations. This will include, in particular, proposing a function space framework for open loop infinite time horizon nonlinear optimal control problems. This topic has received very little attention in the literature even in cases of smooth cost functionals.

To demonstrate the sparsity nature of optimal controls for stabilization problems we consider

$$
\frac{\partial y}{\partial t}-\Delta y+f(y)=u \chi_{\omega}
$$

together with an ininital condition and homogenous Neumann boundary conditions. The nonlinearity $f: \mathbb{R} \rightarrow \mathbb{R}$ is a polynomial function of degree $2 m+1$ with $m \in \mathbb{N}$ arbitrary if $n \leq 2$ and $m=1$ if $n=3$, and further specifications to be given below. Due to the choice of Neumann boundary conditions every root of $f$ is an equilibrium of the uncontrolled state equation. Successive distinct roots of $f$ alternate between stable and unstable behavior. As described above here we are interested in the behavior of sparse controls in the neighborhood of a stable equilibrium, which after a possible change of variables is assumed to be the origin. Consequently, we make the following assumption on $f$ :

$$
f(0)=0, f^{\prime}(0)>0 \text {, and the leading coefficient of } f \text { is positive. }
$$

It will be shown that this condition guarantees that 0 is a stable equilibrium for our dynamical system; see Theorems 2.5 and 2.6.

Let us point out that reaction diffusion systems of polynomial-type arise in many interesting applications including models in physiology, for instance, in the context of FitzHugh-Nagumo models, which describe the prototype of excitable systems; e.g., see [12], or Schlögl's model which is a canonical example of a chemical reaction system [14]. See [3], [8], [13] for the optimal control of these systems.

We are now prepared to formulate the optimal control problem which will be analyzed in this work:

$$
\min _{u \in U} J(u)=\frac{1}{2} \int_{0}^{\infty} \int_{\Omega} y_{u}^{2} d x d t+\frac{\nu}{2} \int_{0}^{\infty} \int_{\omega} u^{2} d x d t+\alpha \int_{0}^{\infty}\left(\int_{\omega} u^{2} d x\right)^{1 / 2} d t,
$$

where

$$
U=L^{2}\left(0, \infty ; L^{2}(\omega)\right) \cap L^{1}\left(0, \infty ; L^{2}(\omega)\right), \quad \nu>0, \quad \alpha>0 .
$$

Here $y_{u}$ denotes the solution of the following parabolic equation:

$$
\left\{\begin{aligned}
\frac{\partial y}{\partial t}-\Delta y+a y+f(y) & =u \chi_{\omega} & & \text { in } Q=\Omega \times(0, \infty), \\
\partial_{n} y & =0 & & \text { on } \Sigma=\Gamma \times(0, \infty), \\
y(0) & =y_{0} & & \text { in } \Omega,
\end{aligned}\right.
$$

where $\Omega$ is a bounded domain in $\mathbb{R}^{n}, 1 \leq n \leq 3$, with a Lipschitz boundary $\Gamma, \omega$ is a subdomain of $\Omega, \chi_{\omega}$ denotes the characteristic function of $\omega, a \in L^{\infty}(\Omega), 0 \leq a \not \equiv 0$, and $y_{0} \in H^{1}(\Omega)$. For every $u \in U$, the symbol $u \chi_{\omega}$ is defined as follows:

$$
\left(u \chi_{\omega}\right)(x, t)=\left\{\begin{array}{cl}
u(x, t) & \text { if }(x, t) \in \omega \times(0, \infty), \\
0 & \text { otherwise }
\end{array}\right.
$$


Remark 1.1. The assumption $a \not \equiv 0$ has been introduced just for simplicity of the presentation, but it is not necessary. All the results of this paper remain valid if we take $a \equiv 0$. Indeed, from the assumptions on $f$ we know that $f(s)=a_{1} s+$ $\cdots+a_{2 m+1} s^{2 m+1}$ with $a_{1}>0$. Therefore for each $\tilde{a}_{1} \in\left(0, a_{1}\right)$ the polynomial $\tilde{f}(s)=$ $\tilde{a}_{1} s+a_{2} s^{2}+\cdots+a_{2 m+1} s^{2 m+1}$ has the same properties as $f$. Therefore, if $a \equiv 0$ we can set $a=a_{1}-\tilde{a}_{1}$ and replace $f$ by $\tilde{f}$ and all the above assumptions are fulfilled.

Concerning the three terms in the cost functional $J$, the first one reflects the objective of stabilization to 0 . The second one is required for the well-posedness of the control problem, and the last one promotes the temporal sparsity of the optimal controls. We observe that cost $J$ can take the value $\infty$ for some controls $u \in U$, because $y_{u} \notin L^{2}(Q)$. We will say that $u \in U$ is a feasible control if $J(u)<\infty$.

We shall frequently use the following property of $f$ which is a consequence of assumption (1.1) and the fact that the polynomial is of odd degree:

$$
\exists \Lambda>0 \text { such that } f^{\prime}(t) \geq-\Lambda \quad \forall t \in \mathbb{R} .
$$

The plan of this paper is as follows. Section 2 contains the analysis of the controlled state equation in spaces with infinite time horizons. Moreover two theorems are presented which provide sufficient conditions for exponential stability of the uncontrolled trajectories provided that the initial condition is either sufficiently small or it is appropriately located with respect to the roots of the polynomial. In section 3 existence of optimal controls is proved and the sensitivity and adjoint equations are analyzed. Finally section 4 contains the optimality system. It allows us to deduce the sparsity properties of the optimal controls.

2. Analysis of the state equation. We shall denote by $L_{l o c}^{2}\left(0, \infty ; H^{1}(\Omega)\right)$ the space of functions $y$ belonging to $L^{2}\left(0, T ; H^{1}(\Omega)\right)$ for every $0<T<\infty$. Analogously we define $L_{l o c}^{2}\left(0, \infty ; L^{2}(\Omega)\right)$ and $L_{l o c}^{\infty}\left(0, \infty ; L^{2}(\Omega)\right)$.

Definition 2.1. We call y a solution to (1.2) if $y \in L_{l o c}^{2}\left(0, \infty ; H^{1}(\Omega)\right) \cap$ $C\left([0, \infty) ; L^{2}(\Omega)\right), f(y) \in L_{\text {loc }}^{2}\left(0, \infty ; L^{2}(\Omega)\right)$, and for every $T>0$ the restriction of y to $Q_{T}=\Omega \times(0, T)$ satisfies in the usual variational sense the equation

$$
\left\{\begin{aligned}
\frac{\partial y}{\partial t}-\Delta y+a y+f(y) & =u \chi_{\omega} & & \text { in } Q_{T}, \\
\partial_{n} y & =0 & & \text { on } \Sigma_{T}, \\
y(0) & =y_{0} & & \text { in } \Omega ;
\end{aligned}\right.
$$

see, for instance, [11, pages 136-137] or [15, page 108] for the definition of a variational solution (or generalized solution) of (2.1).

We have the following existence and uniqueness result.

TheOREM 2.2. For every $u \in L^{2}\left(0, \infty ; L^{2}(\omega)\right)(1.2)$ has a unique solution. Moreover $y \in H_{l o c}^{1}\left(0, \infty ; L^{2}(\Omega)\right)$ holds.

Proof. This proof cannot rely on the usual techniques for semilinear monotone equations because the right-hand side $u$ is not in any $L^{p}\left(0, T ; L^{q}(\Omega)\right)$ space with $p$ and $q$ large enough so that the corresponding state belongs to $L^{\infty}\left(Q_{T}\right)$. First we make the change of variables according to $z(x, t)=\mathrm{e}^{-\Lambda t} y(x, t)$, where $\Lambda$ is introduced in (1.3). Then the resulting equation for $z$ is given by

$$
\left\{\begin{aligned}
\frac{\partial z}{\partial t}-\Delta z+a z+\tilde{f}(t, z) & =\mathrm{e}^{-\Lambda t} u \chi_{\omega} & & \text { in } Q_{T}, \\
\partial_{n} z & =0 & & \text { on } \Sigma_{T}, \\
z(0) & =y_{0} & & \text { in } \Omega,
\end{aligned}\right.
$$


where

$$
\tilde{f}(t, s)=\mathrm{e}^{-\Lambda t} f\left(\mathrm{e}^{\Lambda t} s\right)+\Lambda s \quad \forall(t, s) \in \mathbb{R}^{2} .
$$

For any positive integer $k$ we denote by $\operatorname{Proj}_{[-k,+k]}(s)$ the projection of a real number $s$ on the interval $[-k,+k]$ and we set $\tilde{f}_{k}(t, s)=\tilde{f}\left(t, \operatorname{Proj}_{[-k,+k]}(s)\right)$. As a consequence of (1.1) and (1.3), we have that $\tilde{f}_{k}(t, 0)=0$ and $\partial_{s} \tilde{f}_{k}(t, s)=f^{\prime}\left(\mathrm{e}^{\Lambda t} s\right)+\Lambda \geq 0$ if $|s|<k$ and $\partial_{s} \tilde{f}_{k}(t, s)=0$ if $|s|>k$. Moreover, $\partial_{s} \tilde{f}_{k}(t, 0)=f^{\prime}(0)+\Lambda>0$ holds. By an application of Schauder's fixed point theorem we can obtain the existence of a solution $z_{k} \in L^{2}\left(0, T ; H^{1}(\Omega)\right) \cap C\left([0, T] ; L^{2}(\Omega)\right)$ of the following equation:

$$
\left\{\begin{aligned}
\frac{\partial z_{k}}{\partial t}-\Delta z_{k}+a z_{k}+\tilde{f}_{k}\left(t, z_{k}\right) & =\mathrm{e}^{-\Lambda t} u \chi_{\omega} & & \text { in } Q_{T}, \\
\partial_{n} z_{k} & =0 & & \text { on } \Sigma_{T}, \\
z_{k}(0) & =y_{0} & & \text { in } \Omega .
\end{aligned}\right.
$$

The uniqueness of $z_{k}$ is a consequence of the monotonicity of $\tilde{f}_{k}$. Because of the regularity of $y_{0} \in H^{1}(\Omega)$ and the fact that $u \in L^{2}\left(0, T ; L^{2}(\omega)\right)$, we know that $z_{k} \in$ $H^{1}\left(0, T ; L^{2}(\Omega)\right)$ as well; see, for instance, [15, Proposition III-2.5]. Multiplying (2.3) by $z_{k}$, integrating by parts in $Q_{T}$, and using that $\tilde{f}_{k}\left(t, z_{k}\right) z_{k} \geq 0$, we get the existence of a constant $C_{a}$ such that for every $t \in[0, T]$

$$
\begin{aligned}
& \frac{1}{2}\left\|z_{k}(t)\right\|_{L^{2}(\Omega)}^{2}+C_{a}\left\|z_{k}\right\|_{L^{2}\left(0, t ; H^{1}(\Omega)\right.}^{2} \\
& \leq \frac{1}{2}\left\|z_{k}(t)\right\|_{L^{2}(\Omega)}^{2}+\int_{0}^{t} \int_{\Omega}\left(\left|\nabla z_{k}\right|^{2}+a z_{k}^{2}\right) d x d s \\
& \leq \int_{0}^{t} \int_{\omega} \mathrm{e}^{-\Lambda s} u z_{k} d x d s+\frac{1}{2}\left\|y_{0}\right\|_{L^{2}(\Omega)}^{2} \leq\|u\|_{L^{2}\left(0, t ; L^{2}(\omega)\right)}\left\|z_{k}\right\|_{L^{2}\left(0, t ; H^{1}(\Omega)\right)} \\
& \quad+\frac{1}{2}\left\|y_{0}\right\|_{L^{2}(\Omega)}^{2} \leq \frac{1}{2 C_{a}}\|u\|_{L^{2}\left(0, t ; L^{2}(\omega)\right)}^{2}+\frac{C_{a}}{2}\left\|z_{k}\right\|_{L^{2}\left(0, t ; H^{1}(\Omega)\right)}^{2}+\frac{1}{2}\left\|y_{0}\right\|_{L^{2}(\Omega)}^{2} .
\end{aligned}
$$

From here we deduce

$$
\left\|z_{k}\right\|_{L^{\infty}\left(0, T ; L^{2}(\Omega)\right)}+\left\|z_{k}\right\|_{L^{2}\left(0, T ; H^{1}(\Omega)\right)} \leq \sqrt{\frac{2}{C_{a}}}\|u\|_{L^{2}\left(0, T ; L^{2}(\omega)\right)}+\sqrt{2}\left\|y_{0}\right\|_{L^{2}(\Omega)} .
$$

Next we prove that $\left\{\tilde{f}_{k}\left(\cdot, z_{k}\right)\right\}_{k=1}^{\infty}$ is a bounded sequence in $L^{2}\left(Q_{T}\right)$. Since $\tilde{f}(t, s)$ is a polynomial in the variable $s$ of degree $2 m+1$ with coefficients depending on $t$, but uniformly bounded in $[0, T]$, and leading positive coefficient, elementary calculus leads to the existence of two constants $C_{1}>0$ and $C_{2} \geq 0$ such that

$$
\tilde{f}(t, s)^{2} \leq C_{1} \tilde{f}(t, s) s^{2 m+1}+C_{2} \quad \forall(t, s) \in[0, T] \times \mathbb{R} .
$$

Now, setting $\hat{z}_{k}=\operatorname{Proj}_{[-k,+k]}\left(z_{k}\right)$ we have that $\tilde{f}_{k}\left(t, z_{k}\right)=\tilde{f}\left(t, \hat{z}_{k}\right)$ and

$$
\tilde{f}_{k}\left(t, z_{k}\right)^{2} \leq C_{1} \tilde{f}\left(t, \hat{z}_{k}\right) \hat{z}_{k}^{2 m+1}+C_{2}=C_{1} \tilde{f}_{k}\left(t, z_{k}\right) \hat{z}_{k}^{2 m+1}+C_{2} \quad \forall t \in[0, T] .
$$

Since the sign of the functions $\hat{z}_{k}$ coincide with the sign of $z_{k}$ we get $\tilde{f}_{k}\left(t, z_{k}\right) \hat{z}_{k}^{2 m+1} \geq 0$. Hence the boundedness of $\left\{\tilde{f}_{k}\left(\cdot, z_{k}\right)\right\}_{k=1}^{\infty}$ in $L^{2}\left(Q_{T}\right)$ will follow from the boundedness of $\left\{\tilde{f}_{k}\left(\cdot, z_{k}\right) \hat{z}_{k}^{2 m+1}\right\}_{k=1}^{\infty}$ in $L^{1}\left(Q_{T}\right)$. To prove this boundedness first we observe that $\hat{z}_{k}^{2 m+1} \in H^{1}\left(0, T ; L^{2}(\Omega)\right) \cap L^{2}\left(0, T ; H^{1}(\Omega)\right)$ because $z_{k}$ belongs to this space and 
$\hat{z}_{k} \in L^{\infty}\left(Q_{T}\right)$. Then we can multiply the state equation (2.3) by $\hat{z}_{k}^{2 m+1}$ and integrate by parts. To this end we observe that

$$
\begin{aligned}
& \int_{0}^{T} \int_{\Omega} \frac{\partial z_{k}}{\partial t} \hat{z}_{k}^{2 m+1} d x d t \\
& \quad=\int_{\Omega} z_{k}(T) \hat{z}_{k}^{2 m+1}(T) d x-\int_{\Omega} y_{0} y_{0 k}^{2 m+1} d x-(2 m+1) \int_{0}^{T} \int_{\Omega} z_{k} \frac{\partial \hat{z}_{k}}{\partial t} \hat{z}_{k}^{2 m} d x d t \\
& \quad \geq \int_{\Omega} \hat{z}_{k}^{2 m+2}(T) d x-\int_{\Omega} y_{0}^{2 m+2} d x-(2 m+1) \int_{0}^{T} \int_{\Omega} \frac{\partial \hat{z}_{k}}{\partial t} \hat{z}_{k}^{2 m+1} d x d t,
\end{aligned}
$$

where $y_{0 k}=\operatorname{Proj}_{[-k,+k]}\left(y_{0}\right)$. Recall that $y_{0} \in H^{1}(\Omega) \subset L^{2 m+2}(\Omega)$ for any positive integer $m$ if $n \leq 2$ and for $m \leq 2$ if $n=3$ and so the above integral involving $y_{0}$ is finite. The last integral can be computed as follows:

$$
\begin{aligned}
& \int_{0}^{T} \int_{\Omega} \frac{\partial \hat{z}_{k}}{\partial t} \hat{z}_{k}^{2 m+1} d x d t=\frac{1}{2 m+2} \int_{0}^{T} \frac{d}{d t} \int_{\Omega} \hat{z}_{k}^{2 m+2} d x d t \\
& =\frac{1}{2 m+2}\left(\int_{\Omega} \hat{z}_{k}^{2 m+2}(T) d x-\int_{\Omega} y_{0 k}^{2 m+2} d x\right) .
\end{aligned}
$$

Inserting this identity in (2.5) we get

$$
\int_{0}^{T} \int_{\Omega} \frac{\partial z_{k}}{\partial t} \hat{z}_{k}^{2 m+1} d x d t \geq \frac{1}{2 m+2} \int_{\Omega} \hat{z}_{k}^{2 m+2}(T) d x-\int_{\Omega} y_{0}^{2 m+2} d x .
$$

Moreover we have that $\nabla z_{k} \nabla \hat{z}_{k}^{2 m+1}=(2 m+1) \hat{z}_{k}^{2 m} \nabla z_{k} \nabla \hat{z}_{k}=(2 m+1) \hat{z}_{k}^{2 m}\left|\nabla \hat{z}_{k}\right|^{2}$. Hence combining these facts, we deduce from $(2.3)$

$$
\begin{aligned}
& \frac{1}{2 m+2} \int_{\Omega} \hat{z}_{k}^{2 m+2}(T) d x+\int_{Q_{T}} \hat{z}_{k}^{2 m}\left[(2 m+1)\left|\nabla \hat{z}_{k}\right|^{2}+a \hat{z}_{k}^{2}\right] d x d t \\
& \quad+\int_{Q_{T}} \tilde{f}_{k}\left(t, z_{k}\right) \hat{z}_{k}^{2 m+1} d x d t \leq \int_{0}^{T} \int_{\omega} \mathrm{e}^{-\Lambda t} u \hat{z}_{k}^{2 m+1} d x d t+\int_{\Omega} y_{0}^{2 m+2} d x \\
& \leq\|u\|_{L^{2}\left(0, T ; L^{2}(\omega)\right)}\left(\int_{Q_{T}} \hat{z}_{k}^{4 m+2} d x d t\right)^{1 / 2}+C\left\|y_{0}\right\|_{H^{1}(\Omega)}^{2 m+2} .
\end{aligned}
$$

Since $\tilde{f}(t, s) s^{2 m+1}$ is a polynomial in $s$ of even degree $4 m+2$ and positive leading coefficient, there exist constants $C_{3}>0$ and $C_{4} \leq 0$ such that $\tilde{f}(t, s) s^{2 m+1} \geq C_{3} s^{4 m+2}+C_{4}$. Hence we have that $\tilde{f}_{k}\left(t, z_{k}\right) \hat{z}_{k}^{2 m+1}=\tilde{f}\left(t, \hat{z}_{k}\right) \hat{z}_{k}^{2 m+1} \geq C_{3} \hat{z}_{k}^{4 m+2}+C_{4}$. Using this fact in the above inequality we infer with the Young inequality

$$
\begin{aligned}
& \int_{Q_{T}} \tilde{f}_{k}\left(t, z_{k}\right) \hat{z}_{k}^{2 m+1} d x d t \\
& \quad \leq\|u\|_{L^{2}\left(0, T ; L^{2}(\omega)\right)}\left(\int_{Q_{T}} \hat{z}_{k}^{4 m+2} d x d t\right)^{1 / 2}+C\left\|y_{0}\right\|_{H^{1}(\Omega)}^{2 m+2} \\
& \quad \leq \frac{1}{2 C_{3}}\|u\|_{L^{2}\left(0, T ; L^{2}(\omega)\right)}^{2}+\frac{C_{3}}{2} \int_{Q_{T}} \hat{z}_{k}^{4 m+2} d x d t+C\left\|y_{0}\right\|_{H^{1}(\Omega)}^{2 m+2} \\
& \quad \leq \frac{1}{2 C_{3}}\|u\|_{L^{2}\left(0, T ; L^{2}(\omega)\right)}^{2}+\frac{1}{2} \int_{Q_{T}} \tilde{f}_{k}\left(t, z_{k}\right) \hat{z}_{k}^{2 m+1} d x d t-\frac{C_{4}\left|Q_{T}\right|}{2}+C\left\|y_{0}\right\|_{H^{1}(\Omega)}^{2 m+2},
\end{aligned}
$$


where $\left|Q_{T}\right|$ denotes the Lebesgue measure of $Q_{T}$ and, hence,

$$
\int_{Q_{T}} \tilde{f}_{k}\left(t, z_{k}\right) \hat{z}_{k}^{2 m+1} d x d t \leq \frac{1}{C_{3}}\|u\|_{L^{2}\left(0, T ; L^{2}(\omega)\right)}^{2}-C_{4}\left|Q_{T}\right|+2 C\left\|y_{0}\right\|_{H^{1}(\Omega)}^{2 m+2} .
$$

Thus we have the boundedness of $\left\{\tilde{f}_{k}\left(\cdot, z_{k}\right)\right\}_{k=1}^{\infty}$ in $L^{2}\left(Q_{T}\right)$ as desired. Now using (2.3) we deduce that $\left\{\frac{\partial z_{k}}{\partial t}-\Delta z_{k}\right\}_{k=1}^{\infty}$ is bounded in $L^{2}\left(Q_{T}\right)$. Since $y_{0} \in H^{1}(\Omega)$, from the maximal parabolic regularity property of the heat equation it follows that $\left\{\frac{\partial z_{k}}{\partial t}\right\}_{k=1}^{\infty}$ is bounded in $L^{2}\left(Q_{T}\right)$ as well. Combining this fact with (2.4), we deduce the existence of a subsequence such that

$$
z_{k} \rightarrow z \quad \text { in } L^{2}\left(0, T ; H^{1}(\Omega)\right) \text { and } \quad \frac{\partial z_{k}}{\partial t} \rightarrow \frac{\partial z}{\partial t} \quad \text { in } L^{2}\left(Q_{T}\right),
$$

which implies the strong convergence $z_{k} \rightarrow z$ in $L^{2}\left(Q_{T}\right)$. By taking a new subsequence, if necessary, we can assume that $z_{k}(x, t) \rightarrow z(x, t)$ for almost every point $(x, t) \in Q_{T}$. This implies the pointwise convergence $\tilde{f}_{k}\left(t, z_{k}(x, t)\right) \rightarrow \tilde{f}(t, z(x, t))$ for almost all $(x, t) \in Q_{T}$. This together with the boundedness of $\left\{\tilde{f}_{k}\left(\cdot, z_{k}\right)\right\}_{k=1}^{\infty}$ in $L^{2}\left(Q_{T}\right)$ implies that $f(\cdot, z) \in L^{2}\left(Q_{T}\right)$ and $f_{k}\left(\cdot, z_{k}\right) \rightarrow f(\cdot, z)$ in $L^{2}\left(Q_{T}\right)$. Moreover, since the space $L^{2}\left(0, T ; H^{1}(\Omega)\right) \cap H^{1}\left(0, T ; L^{2}(\Omega)\right.$ is continuously embedded in $C\left([0, T] ; L^{2}(\Omega)\right)$, we deduce that $z$ is in this space. According to the convergence properties of $\left\{z_{k}\right\}_{k=1}^{\infty}$ described above, it is immediate to pass to the limit in (2.3) and deduce that $z$ is a solution of (2.2). Its uniqueness follows from the monotonicity of $\tilde{f}$. From the equivalence between (2.1) and (2.2), we get that $y=\mathrm{e}^{\Lambda t} z$ is the unique solution of (2.1). Since $T$ was an arbitrary positive number we conclude that $y$ satisfies all the requirements of Definition 2.1, and therefore $y$ is the unique solution of (1.2).

Finally, the fact that $y \in H^{1}\left(0, T ; L^{2}(\Omega)\right)$ follows from (2.1) taking into account that $f(y) \in L^{2}\left(Q_{T}\right)$ and $y_{0} \in H^{1}(\Omega)$, [15, Proposition III-2.5].

In the next theorem we establish some infinite horizon regularity properties of the solution of (1.2).

Remark 2.3. We remark that if $y_{0} \in H^{1}(\Omega) \cap L^{\infty}(\Omega)$ and $u \in L^{p}\left(0, T ; L^{q}(\omega)\right)$ with $\frac{1}{p}+\frac{n}{2 q}<1$, then we have that $y \in L^{\infty}\left(Q_{T}\right)$ for every $T>0$. Moreover, if $y_{0} \in C(\bar{\Omega})$, then $y \in C\left(\bar{Q}_{T}\right)$ holds; see, for instance, [11, Chapter III]. The proof is standard for the solution of (2.2) and from here we deduce the corresponding regularity for $y=\mathrm{e}^{\Lambda t} z$.

Theorem 2.4. Let $u \in L^{2}\left(0, \infty ; L^{2}(\omega)\right)$ and let $y$ be the solution of (1.2) corresponding to $u$. If $\|y\|_{L^{2}(Q)}<\infty$, then the following properties hold:

$$
\begin{aligned}
& f(y), y^{2 m+1} \in L^{2}(Q), \\
& y \in L^{2}\left(0, \infty ; H^{1}(\Omega)\right) \cap C\left([0, \infty) ; H^{1}(\Omega)\right) \text { and } \frac{\partial y}{\partial t} \in L^{2}(Q), \\
& \lim _{T \rightarrow \infty}\|y(T)\|_{L^{2}(\Omega)}=0 .
\end{aligned}
$$

Moreover, there exists a constant $C$ independent of $u$ and $y$ such that

$$
\begin{aligned}
& \|f(y)\|_{L^{2}(Q)}+\left\|y^{2 m+1}\right\|_{L^{2}(Q)}+\|y\|_{H^{1}(Q)}+\|y\|_{L^{\infty}\left(0, \infty ; H^{1}(\Omega)\right)} \\
& \quad \leq C\left(\|y\|_{L^{2}(Q)}+\|u\|_{L^{2}\left(0, \infty ; L^{2}(\omega)\right)}+\left\|y_{0}\right\|_{H^{1}(\Omega)}^{m+1}\right) .
\end{aligned}
$$

Proof. We divide the proof into three parts. 
Proof of (2.6). First we demonstrate that $f(y) y^{2 m+1} \in L^{1}(Q)$. Let us write

$$
f(s)=\sum_{j=1}^{2 m+1} a_{j} s^{j} \text { and } C_{f}=\sum_{j=1}^{2 m+1}\left|a_{j}\right| .
$$

Observe that $f(0)=0$ implies that $a_{0}=0$. From here we infer

$$
\left|f(s) s^{2 m+1}\right| \leq C_{f} s^{4 m+2} \forall|s| \geq 1 \text { and }\left|f(s) s^{2 m+1}\right| \leq C_{f} s^{2} \quad \forall|s| \leq 1 .
$$

Moreover, from the properties assumed for $f$ we deduce the existence of $\mu_{0}>0$ and $M_{0}>\mu_{0}$ such that

$$
f^{\prime}(s)>0 \text { and } f(s) s \geq 0 \quad \forall|s| \leq \mu_{0} \text { and } \forall|s| \geq M_{0} .
$$

We will take

$$
\mu=\min \left\{1, \mu_{0}\right\} \text { and } M>\max \left\{M_{0}, \frac{2}{a_{2 m+1}} \sum_{j=1}^{2 m}\left|a_{j}\right|\right\} .
$$

Let us denote $Q^{\delta}=\{(x, t) \in Q:|y(x, t)|>\delta\}$ for any real number $\delta>0$, and $Q^{\mu, M}=\{(x, t) \in Q: \mu<|y(x, t)|<M\}$. Since $y \in L^{2}(Q)$ we have

$$
\left|Q^{\delta}\right| \leq \frac{1}{\delta^{2}} \int_{Q^{\delta}} y^{2}(x, t) d x d t \leq \frac{1}{\delta^{2}}\|y\|_{L^{2}(Q)}^{2}<\infty \quad \forall \delta>0,
$$

hence, $\left|Q^{\mu, M}\right| \leq\left|Q^{\mu}\right|<\infty$ as well. Now we set $C_{M}=\max _{\mu \leq|s| \leq M}|f(s)|$. With this notation and using (2.12) and (2.11) we get for every $T>0$

$$
\begin{aligned}
& \left|\int_{Q_{T}} f(y) y^{2 m+1} d x d t\right| \\
& \quad \leq C_{f} \int_{Q_{T} \backslash Q^{\mu}} y^{2} d x d t+C_{M} M^{2 m+1}\left|Q_{T} \cap Q^{\mu, M}\right| \\
& \quad+\int_{Q_{T} \cap Q^{M}} f(y) y^{2 m+1} d x d t \leq C_{f}\|y\|_{L^{2}(Q)}^{2}+C_{M} M^{2 m+1}\left|Q^{\mu, M}\right| \\
& \quad+\int_{Q_{T} \cap Q^{M}} f(y) y^{2 m+1} d x d t .
\end{aligned}
$$

Thus we only need to prove the integrability of $f(y) y^{2 m+1}$ in $Q^{M}$. To this end, for every $k>M$ we define the projection $y_{k}=\operatorname{Proj}_{[-k,+k]}(y)$ and we multiply (2.1) by $y_{k}^{2 m+1}$ :

$$
\begin{aligned}
\int_{Q_{T}} & \frac{\partial y}{\partial t} y_{k}^{2 m+1} d x d t+\int_{Q}\left[\nabla y \nabla y_{k}^{2 m+1}+a y y_{k}^{2 m+1}\right] d x d t \\
& +\int_{Q_{T} \cap Q^{M}} f(y) y_{k}^{2 m+1} d x d t \\
\leq & C_{f}\|y\|_{L^{2}(Q)}^{2 m}+C_{M} M^{2 m+1}\left|Q^{\mu, M}\right|+\int_{0}^{T} \int_{\omega} u y_{k}^{2 m+1} d x d t .
\end{aligned}
$$


Arguing as in the proof of Theorem 2.2 we have

$$
\int_{0}^{T} \int_{\Omega} \frac{\partial y}{\partial t} y_{k}^{2 m+1} d x d t \geq \frac{1}{2 m+2} \int_{\Omega} y_{k}^{2 m+2}(T) d x-\int_{\Omega} y_{0}^{2 m+2} d x
$$

and $\nabla y \nabla y_{k}^{2 m+1}=(2 m+1) y_{k}^{2 m} \nabla y \nabla y_{k}=(2 m+1) y_{k}^{2 m}\left|\nabla y_{k}\right|^{2}$. Using this in (2.15) and taking into account that (2.12) implies that $f(y(x, t)) y_{k}(x, t) \geq 0$ for every $(x, t) \in$ $Q \backslash Q^{\mu, M}$ we obtain

$$
\begin{aligned}
0 \leq & \int_{Q_{T} \cap Q^{M}} f(y) y_{k}^{2 m+1} d x d t \\
\leq & \int_{\Omega} y_{0}^{2 m+2} d x+C_{f}\|y\|_{L^{2}(Q)}^{2}+C_{M} M^{2 m+1}\left|Q^{\mu, M}\right| \\
& +\|u\|_{L^{2}\left(0,+\infty ; L^{2}(\omega)\right)}\left(\int_{Q_{T} \cap Q^{M}} y_{k}^{4 m+2} d x d t\right)^{1 / 2} \\
\leq & \left\|y_{0}\right\|_{H^{1}(\Omega)}^{2 m+2}+C_{f}\|y\|_{L^{2}(Q)}^{2}+C_{M} M^{2 m+1}\left|Q^{\mu, M}\right| \\
& +\frac{1}{a_{2 m+1}}\|u\|_{L^{2}\left(0,+\infty ; L^{2}(\omega)\right)}^{2}+\frac{a_{2 m+1}}{4} \int_{Q_{T} \cap Q^{M}} y_{k}^{4 m+2} d x d t .
\end{aligned}
$$

Due to the choice of $M$ we have for $|s| \geq M$

$$
\begin{aligned}
f(s) s^{2 m+1} & \geq s^{4 m+2}\left(a_{2 m+1}-\sum_{j=1}^{2 m}\left|a_{j}\right| \frac{1}{|s|^{2 m+1-j}}\right) \\
& \geq s^{4 m+2}\left(a_{2 m+1}-\frac{1}{M} \sum_{j=1}^{2 m}\left|a_{j}\right|\right) \geq \frac{a_{2 m+1}}{2} s^{4 m+2} .
\end{aligned}
$$

Since $f^{\prime}(s)>0$ for $|s| \geq M$ and $k>M$, we get

$$
f(y(x, t)) y_{k}^{2 m+1}(x, t) \geq f\left(y_{k}(x, t)\right) y_{k}^{2 m+1}(x, t) \geq \frac{a_{2 m+1}}{2} y_{k}^{4 m+2}(x, t), \quad(x, t) \in Q^{M} .
$$

Inserting this inequality in the right-hand side of (2.16) and (2.13) we conclude that

$$
\begin{aligned}
0 & \leq \int_{Q_{T} \cap Q^{M}} f(y) y_{k}^{2 m+1} d x d t \\
& \leq C\left(\left\|y_{0}\right\|_{H^{1}(\Omega)}^{2 m+2}+\|y\|_{L^{2}(Q)}^{2}+\|u\|_{L^{2}\left(0, \infty ; L^{2}(\omega)\right)}^{2}\right) \quad \forall T>0 \text { and } \forall k,
\end{aligned}
$$

where $C$ only depends on $f$ and $M$. Since $y_{k}(x, t) \rightarrow y(x, t)$ a.e. in $Q$, we deduce from the above inequality, (2.13), (2.14), and Fatou's lemma that

$$
\int_{Q}\left|f(y) \| y^{2 m+1}\right| d x d t \leq C\left(\left\|y_{0}\right\|_{H^{1}(\Omega)}^{2 m+2}+\|y\|_{L^{2}(Q)}^{2}+\|u\|_{L^{2}\left(0, \infty ; L^{2}(\omega)\right)}^{2}\right)
$$

for a new $C$ only depending on $f$ and $M$. Now we have

$$
\begin{aligned}
\int_{Q} y^{4 m+2} d x d t & \leq \int_{Q \backslash Q^{\mu}} y^{2} d x d t+\int_{Q^{\mu, M}} M^{4 m+2} d x d t+\int_{Q^{M}} y^{4 m+2} d x d t \\
& \leq\|y\|_{L^{2}(Q)}^{2}+M^{4 m+2}\left|Q^{\mu, M}\right|+\frac{2}{a_{2 m+1}} \int_{Q^{M}} f(y) y^{2 m+1} d x d t<\infty,
\end{aligned}
$$


which proves that $y^{2 m+1} \in L^{2}(Q)$. Moreover, since $|f(s)| \leq C_{f}|s|^{2 m+1} \forall|s| \geq 1$ and $|f(s)| \leq C_{f}|s| \forall|s| \leq 1$, we deduce that

$$
f(s)^{2} \leq C_{f}^{2}\left(s^{2}+s^{4 m+2}\right) \quad \forall s \in \mathbb{R} .
$$

Therefore, the fact that $y$ and $y^{2 m+1} \in L^{2}(Q)$ implies that $f(y) \in L^{2}(Q)$ and the proof of (2.6) is complete. Additionally, these arguments obviously lead to the estimates for the first two terms of (2.9).

Proof of (2.7). First we observe that $y \in C\left([0, T] ; H^{1}(\Omega)\right)$ for every $T>0$. Indeed, this is a consequence of the fact that $f(y) \in L^{2}(Q)$ and $y_{0} \in H^{1}(\Omega)$; see [15, Proposition III-2.5]. Hence $y:[0, \infty) \rightarrow H^{1}(\Omega)$ is continuous. To prove that $y \in L^{2}\left(0, \infty ; H^{1}(\Omega)\right)$ it is enough to multiply $(2.1)$ by $y$ and integrate in $Q_{T}, T>0$ arbitrary, to get

$$
\begin{aligned}
& \frac{1}{2}\|y(T)\|_{L^{2}(\Omega)}+\int_{Q_{T}}\left(|\nabla y|^{2}+a y^{2}\right) d x d t \\
& \quad=\int_{0}^{T} \int_{\omega} u y d x d t+\frac{1}{2}\left\|y_{0}\right\|_{L^{2}(\Omega)}^{2}-\int_{Q_{T}} f(y) y d x d t \\
& \quad \leq\|u\|_{L^{2}\left(0, \infty ; L^{2}(\omega)\right)}\|y\|_{L^{2}(Q)}+\frac{1}{2}\left\|y_{0}\right\|_{L^{2}(\Omega)}^{2}+\|f(y)\|_{L^{2}(Q)}\|y\|_{L^{2}(Q)}<\infty
\end{aligned}
$$

Above we have used the assumption $y \in L^{2}(Q)$. Now it is enough to make $T \rightarrow \infty$ to deduce that $y \in L^{2}\left(0, \infty ; H^{1}(\Omega)\right)$.

To prove that $y \in L^{\infty}\left(0, \infty ; H^{1}(\Omega)\right)$ we take into account that by Theorem 2.2 $y \in H^{1}\left(0, T ; L^{2}(\Omega)\right) \cap L^{2}\left(0, T ; H^{1}(\Omega)\right)$ for every $T>0$. We can multiply (2.1) by $\frac{\partial y}{\partial t}$ and integrate in $Q_{T}$ to get

$$
\left\|\frac{\partial y}{\partial t}\right\|_{L^{2}\left(Q_{T}\right)}^{2}+\int_{0}^{T} \frac{1}{2} \frac{d}{d t} \int_{\Omega}\left(|\nabla y|^{2}+a y^{2}\right) d x+\int_{Q_{T}} f(y) \frac{\partial y}{\partial t} d x d t=\int_{0}^{T} \int_{\omega} u \frac{\partial y}{\partial t} d x d t .
$$

This implies

$$
\begin{aligned}
& \left\|\frac{\partial y}{\partial t}\right\|_{L^{2}\left(Q_{T}\right)}^{2}+\frac{1}{2} \int_{\Omega}\left(|\nabla y(T)|^{2}+a_{0} y^{2}(T)\right) d x \\
& \quad \leq\left(\|u\|_{L^{2}\left(0, T ; L^{2}(\omega)\right)}+\|f(y)\|_{L^{2}(Q)}\right)\left\|\frac{\partial y}{\partial t}\right\|_{L^{2}\left(Q_{T}\right)}+\frac{1}{2} \int_{\Omega}\left(\left|\nabla y_{0}\right|^{2}+a_{0} y_{0}^{2}\right) d x \\
& \quad \leq \frac{1}{2}\left(\|u\|_{L^{2}\left(0, T ; L^{2}(\omega)\right)}+\|f(y)\|_{L^{2}(Q)}\right)^{2}+\frac{1}{2}\left\|\frac{\partial y}{\partial t}\right\|_{L^{2}\left(Q_{T}\right)}^{2}+\frac{C}{2}\left\|y_{0}\right\|_{H^{1}(\Omega)}^{2},
\end{aligned}
$$

hence,

$$
\begin{aligned}
& \left\|\frac{\partial y}{\partial t}\right\|_{L^{2}\left(Q_{T}\right)}^{2}+\int_{\Omega}\left(|\nabla y(T)|^{2}+a_{0} y^{2}(T)\right) d x \\
& \quad \leq\left(\|u\|_{L^{2}\left(0, T ; L^{2}(\omega)\right)}+\|f(y)\|_{L^{2}(Q)}\right)^{2}+C\left\|y_{0}\right\|_{H^{1}(\Omega)}^{2} .
\end{aligned}
$$

Since $T>0$ is arbitrary, the above inequality concludes the proof of (2.7). Moreover, from the obtained estimates the bounds for the second two terms in (2.9) follow.

Proof of (2.8). Since $y \in H^{1}\left(0, T ; L^{2}(\Omega)\right)$ for every $T>0$, then the function $t \in[0, T] \rightarrow\|y(t)\|_{L^{2}(\Omega)}^{2}$ is absolutely continuous and

$$
\frac{d}{d t}\|y(t)\|_{L^{2}(\Omega)}^{2}=2 \int_{\Omega} y(t) \frac{\partial y}{\partial t}(t) d x
$$


see, for instance, [15, Proposition III-1.2]. Moreover, the fact that $y \in L^{2}(Q)$ implies the existence of a monotone increasing sequence of positive numbers $\left\{t_{k}\right\}_{k=1}^{\infty}$ such that $\left\|y\left(t_{k}\right)\right\|_{L^{2}(\Omega)} \rightarrow 0$ as $k \rightarrow \infty$. Then, given $T>0$ and taking $t_{k}>T$ we get

$$
\begin{aligned}
\|y(T)\|_{L^{2}(\Omega)}^{2} & =\left\|y\left(t_{k}\right)\right\|_{L^{2}(\Omega)}^{2}-2 \int_{T}^{t_{k}} \int_{\Omega} y(t) \frac{\partial y}{\partial t}(t) d x d t \\
& \leq\left\|y\left(t_{k}\right)\right\|_{L^{2}(\Omega)}^{2}+2\|y\|_{L^{2}\left(T, \infty ; L^{2}(\Omega)\right)}\left\|\frac{\partial y}{\partial t}\right\|_{L^{2}\left(T, \infty ; L^{2}(\Omega)\right)} .
\end{aligned}
$$

Taking the limit when $k \rightarrow \infty$ we get

$$
\|y(T)\|_{L^{2}(\Omega)}^{2} \leq \lim _{T \rightarrow \infty} 2\|y\|_{L^{2}\left(T, \infty ; L^{2}(\Omega)\right)}\left\|\frac{\partial y}{\partial t}\right\|_{L^{2}\left(T, \infty ; L^{2}(\Omega)\right)}=0 .
$$

Theorem 2.4 suggests introducing the following space of solutions:

$$
Y=\left\{y \in H^{1}(Q) \cap B C\left([0, \infty) ; H^{1}(\Omega)\right): \Delta y \in L^{2}(Q) \text { and } \partial_{n} y=0 \text { on } \Sigma\right\},
$$

where $B C\left([0, \infty) ; H^{1}(\Omega)\right)$ is the Banach space of continuous and bounded functions $y:[0, \infty) \longrightarrow H^{1}(\Omega)$. The space $Y$ endowed with the norm

$$
\|y\|_{Y}=\|y\|_{H^{1}(Q)}+\|y\|_{L^{\infty}\left(0, \infty, H^{1}(\Omega)\right)}+\|\Delta y\|_{L^{2}(Q)}
$$

is a Banach space. Let us point out that any element $y \in H^{1}\left(0, \infty ; L^{2}(\Omega)\right)$ satisfies (2.8). This was proved in the last step of the proof of Theorem 2.4. Hence this property holds for every element $y \in Y$.

In the next theorem we prove that if $\left\|y_{0}\right\|_{L^{2}(\Omega)}$ is sufficiently small, then the solution of (1.2) associated with the null control $u \equiv 0$ is stable and it has an exponential decay. Let us introduce some notation to make precise how small $\left\|y_{0}\right\|_{L^{2}(\Omega)}$ must be. Let $\mu_{0}$ and $M_{0}$ satisfy (2.12) and set $C_{0}^{\prime}=\max _{\mu_{0} \leq|s| \leq M_{0}}\left|f^{\prime}(s)\right|$. Now we take

$$
K_{f}=\frac{\mu_{0} C_{a}^{2}}{\left(C_{0}^{\prime} C_{4}\right)^{2}}
$$

where $C_{a}>0$ and $C_{4}>0$ are taken so that

$$
\begin{aligned}
\int_{\Omega}\left(|\nabla z|^{2}+a z^{2}\right) d x & \geq C_{a}\|z\|_{H^{1}(\Omega)}^{2} \geq C_{a}\|z\|_{L^{2}(\Omega)}^{2}, \\
\|z\|_{L^{4}(\Omega)} & \leq C_{4}\|z\|_{H^{1}(\Omega)} \quad \forall z \in H^{1}(\Omega) .
\end{aligned}
$$

THEOREM 2.5. Let us assume that $\left\|y_{0}\right\|_{L^{2}(\Omega)}<K_{f}$ and $u \equiv 0$. Then the solution of (1.2) belongs to $L^{2}(Q)$ and there exists $\lambda>0$ such that

$$
\|y(t)\|_{L^{2}(\Omega)} \leq\left\|y_{0}\right\|_{L^{2}(\Omega)} e^{-\lambda t} \quad \forall t>0 .
$$

Together with (2.9) of Theorem 2.4 this theorem provides a sufficient condition for $y \in Y$.

Proof. Let us take

$$
K_{0}=\frac{1}{2}\left(\left\|y_{0}\right\|_{L^{2}(\Omega)}+K_{f}\right) \text { and } T_{0}=\sup \left\{T>0:\|y(t)\|_{L^{2}(\Omega)}<K_{0}, \forall t \in[0, T]\right\} .
$$


Since $y:[0, \infty) \rightarrow L^{2}(\Omega)$ is a continuous function and $\|y(0)\|_{L^{2}(\Omega)}=\left\|y_{0}\right\|_{L^{2}(\Omega)}<K_{0}$, we have that $T_{0}>0$. We will prove that $T_{0}=\infty$. For every $t \in\left(0, T_{0}\right)$ we define

$$
\Omega_{t}=\left\{x \in \Omega: \mu_{0}<|y(x, t)|<M_{0}\right\}
$$

Then we have

$$
\left|\Omega_{t}\right| \leq \frac{1}{\mu_{0}^{2}} \int_{\Omega_{t}} y^{2}(t) d x \leq \frac{1}{\mu_{0}^{2}}\|y(t)\|_{L^{2}(\Omega)}^{2}<\frac{K_{0}^{2}}{\mu_{0}^{2}} \quad \forall t \in\left(0, T_{0}\right) .
$$

We will use the following interpolation inequality (see, e.g., [4, p. 93]):

$$
\|z\|_{L^{8 / 3}(\Omega)} \leq\|z\|_{L^{2}(\Omega)}^{1 / 2}\|z\|_{L^{4}(\Omega)}^{1 / 2} \leq C_{4}^{1 / 2}\|z\|_{L^{2}(\Omega)}^{1 / 2}\|z\|_{H^{1}(\Omega)}^{1 / 2} \quad \forall z \in H^{1}(\Omega) .
$$

Then, multiplying (1.2) by $y(t), t \in\left(0, T_{0}\right)$, integrating in $\Omega$, taking into account that $f(y(t)) y(t) \geq 0$ in $\Omega \backslash \Omega_{t}$ by (2.12), using (2.20) and (2.21), and Young's inequality we deduce for almost all $t \in\left(0, T_{0}\right)$ for some $\theta(x, t) \in[0,1]$

$$
\begin{aligned}
& \frac{1}{2} \frac{d}{d t}\|y(t)\|_{L^{2}(\Omega)}^{2}+C_{a}\|y(t)\|_{H^{1}(\Omega)}^{2} \\
& \leq \frac{1}{2} \frac{d}{d t}\|y(t)\|_{L^{2}(\Omega)}^{2}+\int_{\Omega}\left(|\nabla y(t)|^{2}+a y(t)^{2}\right) d x \\
&\left.\quad+\int_{\Omega \backslash \Omega_{t}} f(y(t)) y(t) d x=-\int_{\Omega_{t}} f(y(t)) y(t)\right) d x=-\int_{\Omega_{t}} f^{\prime}(\theta(t) y(t)) y^{2}(t) d x \\
& \leq\left\|f^{\prime}(\theta(t) y(t))\right\|_{L^{4}\left(\Omega_{t}\right)}\|y(t)\|_{L^{8 / 3}(\Omega)}^{2} \leq C_{0}^{\prime}\left|\Omega_{t}\right|^{1 / 4} C_{4}\|y(t)\|_{L^{2}(\Omega)}\|y(t)\|_{H^{1}(\Omega)} \\
& \leq \frac{C_{0}^{\prime 2} C_{4}^{2}\left|\Omega_{t}\right|^{1 / 2}}{2 C_{a}}\|y(t)\|_{L^{2}(\Omega)}^{2}+\frac{C_{a}}{2}\|y(t)\|_{H^{1}(\Omega)}^{2} .
\end{aligned}
$$

With (2.20) this leads to

$$
\begin{aligned}
& \frac{1}{2} \frac{d}{d t}\|y(t)\|_{L^{2}(\Omega)}^{2}+\frac{C_{a}}{2}\|y(t)\|_{L^{2}(\Omega)}^{2} \leq \frac{1}{2} \frac{d}{d t}\|y(t)\|_{L^{2}(\Omega)}^{2}+\frac{C_{a}}{2}\|y(t)\|_{H^{1}(\Omega)}^{2} \\
& \quad \leq \frac{C_{0}^{\prime 2} C_{4}^{2}\left|\Omega_{t}\right|^{1 / 2}}{2 C_{a}}\|y(t)\|_{L^{2}(\Omega)}^{2} \leq \frac{C_{0}^{\prime 2} C_{4}^{2} K_{0}}{2 C_{a} \mu_{0}}\|y(t)\|_{L^{2}(\Omega)}^{2},
\end{aligned}
$$

hence,

$$
\frac{1}{2} \frac{d}{d t}\|y(t)\|_{L^{2}(\Omega)}^{2}+\lambda\|y(t)\|_{L^{2}(\Omega)}^{2} \leq 0
$$

where

$$
\lambda=\frac{1}{2}\left(C_{a}-\frac{C_{0}^{\prime 2} C_{4}^{2} K_{0}}{C_{a} \mu_{0}}\right) .
$$

From the choice of $K_{0}$ and (2.17) we infer that $\lambda>0$. Then we have $\frac{d}{d t}\|y(t)\|_{L^{2}(\Omega)}^{2} \leq 0$ $\forall t \in\left(0, T_{0}\right)$, hence, $T_{0}=\infty$. Moreover, inequality (2.22) implies (2.19).

Let us denote by $\rho_{-}$the biggest negative root of the polynomial $f$ such that $f(\rho)$ changes sign when $\rho$ crosses $\rho_{-}$, i.e., $\rho_{-}$is a root of $f$ of odd multiplicity. If $f$ has no negative root with such a property, then we set $\rho_{-}=-\infty$. Analogously, we define $\rho_{+}$as the smallest positive root of $f$ with odd multiplicity, and we take $\rho_{+}=+\infty$ if such a root does not exist. Then we have the following theorem. 
THEOREM 2.6. Let us assume that $\rho_{-} \leq y_{0}(x) \leq \rho_{+}$for a.a. $x \in \Omega$, and $u \equiv 0$. Then the solution $y$ of (1.2) belongs to $L^{2}(Q), \rho_{-} \leq y(x, t) \leq \rho_{+} \forall(x, t) \in Q$, and

$$
\|y(t)\|_{L^{2}(\Omega)} \leq\left\|y_{0}\right\|_{L^{2}(\Omega)} e^{-C_{a} t} \quad \forall t>0,
$$

where $C_{a}>0$ is given by (2.18)

Proof. First we assume that $y_{0} \in C(\bar{\Omega})$ and $\rho_{-}<y_{0}(x)<\rho_{+} \forall x \in \bar{\Omega}$. Let us set $\lambda_{-}=\min \left\{0, \min _{x \in \bar{\Omega}} y_{0}(x)\right\}$ and $\lambda_{+}=\max \left\{0, \max _{x \in \bar{\Omega}} y_{0}(x)\right\}$. Then we have $\rho_{-}<\lambda_{-} \leq 0 \leq \lambda_{+}<\rho_{+}$. Let $y$ be the solution of (1.2) associated with $u \equiv 0$. Since $y_{0} \in C(\bar{\Omega})$, then $y \in C\left(\bar{Q}_{T}\right) \forall T>0$; see Remark 2.3. Then, $\lim _{t \rightarrow 0}\left\|y(t)-y_{0}\right\|_{C(\bar{\Omega})}=0$ holds. This implies that

$$
T^{*}=\sup \left\{T>0: \rho_{-}<y(x, t)<\rho_{+} \forall(x, t) \in \bar{Q}_{T}\right\}>0 .
$$

Let us prove that $T^{*}=+\infty$. We argue by contradiction. If $T^{*}<+\infty$, then there exists at least one point $x^{*} \in \bar{\Omega}$ such that $\rho_{-}<y(x, t)<\rho_{+} \forall(x, t) \in \bar{\Omega} \times\left[0, T^{*}\right)$ and either $y\left(x^{*}, T^{*}\right)=\rho_{-}$or $y\left(x^{*}, T^{*}\right)=\rho_{+}$. Let us assume that $y\left(x^{*}, T^{*}\right)=\rho_{-}$ and take $z(x, t)=\left(y(x, t)-\lambda_{-}\right)^{-}=\min \left\{y(x, t)-\lambda_{-}, 0\right\}$. It is clear that $\frac{\partial y}{\partial t} z=\frac{\partial z}{\partial t} z$, $\nabla y \nabla z=|\nabla z|^{2}$, and $y z \geq z^{2}$ a.e., in $Q_{T^{*}}$. Hence, multiplying (2.1) by $z$, integrating in $Q_{T^{*}}$, and using that $z(0)=0$ we get

$$
\left\|z\left(T^{*}\right)\right\|_{L^{2}(\Omega)}^{2}+\int_{Q_{T^{*}}}\left(|\nabla z|^{2}+a z^{2}\right) d x d t+\int_{Q_{T^{*}}} f(y) z d x d t=0 .
$$

Now, we observe that $f(s) s \geq 0 \forall s \in\left[\rho_{-}, \rho_{+}\right]$, hence, $f(y(x, t)) z(x, t) \geq 0 \forall(x, t) \in$ $Q_{T^{*}}$. Using this in the above inequality we obtain with (2.18)

$$
\left\|z\left(T^{*}\right)\right\|_{L^{2}(\Omega)}^{2}+C_{a}\|z\|_{L^{2}\left(Q_{T^{*}}\right)}^{2} \leq 0,
$$

and, therefore, $z=0$. This implies that $y(x, t) \geq \lambda_{-}>\rho_{-} \forall(x, t) \in Q_{T^{*}}$, which contradicts the equality $y\left(x^{*}, T^{*}\right)=\rho_{-}$.

In the case $y\left(x^{*}, t^{*}\right)=\rho_{+}$, we take $z(x, t)=\max \left\{y(x, t)-\lambda_{+}, 0\right\}$ and we argue similarly as above. Hence $T^{*}=\infty$ holds. If $\lambda_{-}=\rho_{-}$or $\lambda_{+}=\rho_{+}$, then we take $y_{0 \varepsilon}=\operatorname{Proj}_{\left[\lambda_{-}+\varepsilon, \lambda_{+}-\varepsilon\right]}\left(y_{0}(x)\right)$. Then $y_{0 \varepsilon} \rightarrow y_{0}$ in $C(\bar{\Omega}) \cap H^{1}(\Omega)$ holds. If we denote by $y_{\varepsilon}$ the solution of (1.2) associated with $y_{0 \varepsilon}$ and $u \equiv 0$, then we have that $y_{\varepsilon} \rightarrow y$ in $\bar{Q}_{T}$ for every $T>0$. Since $\rho_{-} \leq y_{\varepsilon}(x, t) \leq \rho_{+} \forall(x, t) \in Q$, we conclude that $\rho_{-} \leq y(x, t) \leq \rho_{+} \forall(x, t) \in Q$ as well.

Since $f(y(x, t)) y(x, t) \geq 0 \forall(x, t) \in Q$, multiplying (1.2) by $y$ and integrating in $\Omega$ we get

$$
\frac{1}{2} \frac{d}{d t}\|y(t)\|_{L^{2}(\Omega)}^{2}+C_{a}\|y(t)\|_{L^{2}(\Omega)}^{2} \leq 0,
$$

which implies (2.23) and, consequently, $y \in L^{2}(Q)$.

Finally, if $y_{0} \in H^{1}(\Omega) \backslash C(\bar{\Omega})$ and $\rho_{-} \leq y_{0}(x) \leq \rho_{+}$for a.a. $x \in \Omega$, then we take a sequence $\left\{z_{k}\right\}_{k=1}^{\infty} \subset H^{1}(\Omega) \cap C(\bar{\Omega})$ such that $z_{k} \rightarrow y_{0}$ in $H^{1}(\Omega)$ and $z_{k}(x) \rightarrow y_{0}(x)$ a.e. in $\Omega$. Now, we take $y_{0 k}(x)=\operatorname{Proj}_{\left[\rho_{-}, \rho_{+}\right]}\left(z_{k}(x)\right)$, and we still have that $\left\{y_{0 k}\right\}_{k=1}^{\infty} \subset$ $H^{1}(\Omega) \cap C(\bar{\Omega}), y_{0 k} \rightarrow y_{0}$ in $H^{1}(\Omega)$, and $y_{0 k}(x) \rightarrow y_{0}(x)$ a.e. in $\Omega$. The solution $y_{k}$ of $(1.2)$ corresponding to the initial condition $y_{0 k}$ belongs to $L^{2}(Q), \rho_{-} \leq y_{k}(x, t) \leq \rho_{+}$ $\forall(x, t) \in Q$, and it satisfies

$$
\left\|y_{k}(t)\right\|_{L^{2}(\Omega)} \leq\left\|y_{0 k}\right\|_{L^{2}(\Omega)} \mathrm{e}^{-C_{a} t} \forall t>0 .
$$

Now, it is easy to prove the boundedness of $\left\{y_{k}\right\}_{k=1}^{\infty}$ in $Y$ and, hence, we pass to the limit in the above inequality as $k \rightarrow \infty$ and we deduce that $y$ satisfies (2.23). 
Remark 2.7. Let us come back to our original equation

$$
\frac{\partial y}{\partial t}-\Delta y+f(y)=u \chi_{\omega}
$$

where $a \equiv 0$. We consider $\rho_{-}$and $\rho_{+}$as in Theorem 2.6. Then the proof of Theorem 2.6 and (2.23) fail. However we can still stabilize the system by a feedback control. Indeed, let us take $a=\lambda \chi_{\omega}$, where $\lambda$ is an arbitrary strictly positive constant. Then we are under the conditions of Theorem 2.6 and the theorem holds. Now, we take $u=-a y=-\lambda \chi_{\omega} y,(2.24)$ holds, and the state $y$ is stabilized.

Remark 2.8. We remark that the fact that the nonlinearity $f$ in (1.2) is a polynomial function played an essential role in the proofs of this section. However, if we assume that $y_{0} \in H^{1}(\Omega) \cap L^{2 m+2}(\Omega)$, then all the results of this section are valid for a polynomial of arbitrary degree $2 m+1$, with the obvious changes in the estimates. The assumption $m=1$ in dimension 3 will be used in Theorem 3.4 below.

3. Analysis of the control problem. We divide this section into three parts. First we study the existence of an optimal control. Then, we address the sensitivity of the states with respect to the controls, and finally we analyze the adjoint state equation.

3.1. Existence of a solution to $(\mathbf{P})$. Before proving the existence of an optimal control we establish the following lemma.

LEMma 3.1. Let $\left\{u_{k}\right\}$ be a bounded sequence in $L^{2}\left(0, \infty ; L^{2}(\omega)\right)$ such that the corresponding states $\left\{y_{k}\right\}$ are bounded in $L^{2}(Q)$. Then, there exist subsequences such that $u_{k} \rightarrow \bar{u}$ in $L^{2}\left(0, \infty ; L^{2}(\omega)\right)$ and $y_{k} \rightarrow \bar{y}$ in $H^{1}(Q)$, where $\bar{y}$ is the state associated with $\bar{u}$.

Proof. From the assumptions of the lemma and (2.9) we deduce the existence of subsequences such that $u_{k} \rightarrow \bar{u}$ in $L^{2}\left(0, \infty ; L^{2}(\omega)\right)$ and $y_{k} \rightarrow \bar{y}$ in $H^{1}(Q)$. We prove that $\bar{y}$ is the solution of (1.2) associated with $\bar{u}$. To this end we have to check Definition 2.1. First we observe that $\bar{y} \in H^{1}(Q) \subset C\left([0, \infty) ; L^{2}(\Omega)\right)$. Now let $T>0$ be arbitrary. From the compactness of the embedding $H^{1}\left(Q_{T}\right) \subset L^{2}\left(Q_{T}\right)$ we infer the existence of a subsequence such that

$$
y_{k} \rightarrow \bar{y} \text { in } L^{2}\left(Q_{T}\right) \text { and } y_{k}(x, t) \rightarrow \bar{y}(x, t) \text { a.e. in } Q_{T} .
$$

Using again (2.9) we deduce from the above pointwise convergence that $f\left(y_{k}\right) \rightarrow f(\bar{y})$ in $L^{2}\left(Q_{T}\right)$. Then it is easy to pass to the limit weakly in the state equation (1.2) and to deduce that $\bar{y}$ satisfies the equation in the variational sense in $Q_{T}$ with $\bar{u}$ on the right-hand side. Moreover, from the continuity of the embedding $H^{1}\left(Q_{T}\right) \subset$ $C\left([0, T] ; L^{2}(\Omega)\right)$ we have $\bar{y}(0)=\lim _{k \rightarrow \infty} y_{k}(0)=y_{0}$.

Theorem 3.2. Assume that there exists an element $u_{0} \in L^{2}\left(0, \infty ; L^{2}(\omega)\right)$ such that $J\left(u_{0}\right)<\infty$. Then $(\mathrm{P})$ admits at least one solution.

Proof. Since the set of feasible controls is nonempty, we can take a minimizing sequence $\left\{u_{k}\right\}$. From the inequality $J\left(u_{k}\right) \leq J\left(u_{0}\right)$ for every $k$ large (unless $u_{0}$ is already an optimal control), we deduce the boundedness of $\left\{\left(u_{k}, y_{k}\right)\right\}$ in $L^{2}\left(0, \infty ; L^{2}(\omega)\right) \cap$ $L^{1}\left(0, \infty, L^{2}(\omega)\right) \times L^{2}(Q)$, where $y_{k}$ denotes the state associated with $u_{k}$. Let $(\bar{u}, \bar{y})$ be a weak limit in $L^{2}\left(0, \infty ; L^{2}(\omega)\right) \times L^{2}(Q)$ of a subsequence, denoted in the same way. Lemma 3.1 implies that $\bar{y}$ is the solution of (1.2) corresponding to $\bar{u}$. To prove that 
$\bar{u}$ is a solution to $(\mathrm{P})$, we consider the following inequality for every $T>0$ :

$\frac{1}{2} \int_{Q_{T}} \bar{y}^{2} d x d t+\frac{\nu}{2} \int_{0}^{T} \int_{\omega} \bar{u}^{2} d x d t+\alpha \int_{0}^{T}\left(\int_{\omega} \bar{u}^{2} d x\right)^{1 / 2} d t \leq \liminf _{k \rightarrow \infty} J\left(u_{k}\right)=\inf (\mathrm{P})$,

which follows from the convexity of the objective functional with respect to pair $(y, u)$ and the continuity of the embedding $L^{2}\left(0, T ; L^{2}(\omega)\right) \subset L^{1}\left(0, T ; L^{2}(\omega)\right)$. Now we have

$J(\bar{u})=\sup _{T>0}\left\{\frac{1}{2} \int_{Q_{T}} \bar{y}^{2} d x d t+\frac{\nu}{2} \int_{0}^{T} \int_{\omega} \bar{u}^{2} d x d t+\alpha \int_{0}^{T}\left(\int_{\omega} \bar{u}^{2} d x\right)^{1 / 2} d t\right\}=\inf (\mathrm{P})$,

which concludes the proof.

Remark 3.3. Concerning the feasibility assumption of Theorem 3.2, Theorems 2.5 and 2.6 provide sufficient conditions on $y_{0}$ to ensure that $u_{0} \equiv 0$ is a feasible control for $(\mathrm{P})$. If $y_{0}$ does not satisfy the required assumptions, but (1.2) is approximately controllable to zero, again we can rely on the above theorems to guarantee existence of a feasible control. In particular, for the case $\omega=\Omega$ we can prove approximate controllability as follows. First we solve the equation

$$
\left\{\begin{aligned}
\frac{\partial y}{\partial t}-\Delta y+(a+\Lambda) y+f(y) & =0 & & \text { in } Q, \\
\partial_{n} y & =0 & & \text { on } \Sigma, \\
y(0) & =y_{0} & & \text { in } \Omega,
\end{aligned}\right.
$$

where $\Lambda$ is given by in (1.3). Then we have

$$
\Lambda s^{2}+f(s) s=\left(\Lambda+f^{\prime}(\theta s)\right) s^{2} \geq 0 \quad \forall s \in \mathbb{R},
$$

where $\theta=\theta(s) \in[0,1]$.

Hence, we infer the estimate

$$
\begin{aligned}
& \frac{1}{2} \frac{d}{d t}\|y(t)\|_{L^{2}(\Omega)}^{2}+C_{a}\|y(t)\|_{L^{2}(\Omega)}^{2} \\
& \quad \leq \frac{1}{2} \frac{d}{d t}\|y(t)\|_{L^{2}(\Omega)}^{2}+\int_{\Omega}\left[|\nabla y(t)|^{2}+(a+\Lambda) y(t)^{2}\right] d x+\int_{\Omega} f(y(t)) y(t) d x=0 .
\end{aligned}
$$

Therefore we can argue as in the proofs of Theorems 2.2 and 2.4 to deduce the existence of a unique solution $y \in Y$. Moreover, from the above inequality we infer

$$
\|y(t)\|_{L^{2}(\Omega)} \leq\left\|y_{0}\right\|_{L^{2}(\Omega)} \mathrm{e}^{-C_{a} t}
$$

Now, it is enough to take $u=-\Lambda y$ in (1.2) and then $y_{u}=y$ and $u \in U$. Indeed, since $Y \subset L^{2}(Q)$ we have that $u \in L^{2}(Q)$. Furthermore from the last inequality we get

$$
\int_{0}^{\infty}\|u(t)\|_{L^{2}(\Omega)} d t \leq \Lambda\left\|y_{0}\right\|_{L^{2}(\Omega)} \int_{0}^{\infty} \mathrm{e}^{-C_{a} t} d t<\infty .
$$

Thus $u$ is a feasible control for $(\mathrm{P})$.

3.2. Sensitivity of the relationship control-to-state. We define $\mathcal{U}$ as the subset of the elements $u \in L^{2}\left(0, \infty ; L^{2}(\omega)\right)$ for which there exists a solution $y_{u} \in Y$ as well as the mapping $G: \mathcal{U} \longrightarrow Y$ by $G(u)=y_{u}$. We remark that for every $u \in L^{2}\left(0, \infty ; L^{2}(\omega)\right)$ there exists a unique solution $y_{u}$ in the sense of Definition 2.1. Further, due to Theorem 2.4, an element $u \in L^{2}\left(0, \infty ; L^{2}(\omega)\right)$ belongs to $\mathcal{U}$ if and only if $y_{u} \in L^{2}(Q)$. 
Theorem 3.4. The set $\mathcal{U}$ is open in $L^{2}\left(0, \infty ; L^{2}(\omega)\right)$ and $G: \mathcal{U} \longrightarrow Y$ is of class $C^{1}$. Furthermore, for every $(u, v) \in \mathcal{U} \times L^{2}\left(0, \infty ; L^{2}(\omega)\right)$ the derivative $z_{v}=D G(u) v$ is the unique solution to

$$
\left\{\begin{aligned}
\frac{\partial y}{\partial t}-\Delta z+a z+f^{\prime}\left(y_{u}\right) z & =v \chi_{\omega} & & \text { in } Q \\
\partial_{n} z & =0 & & \text { on } \Sigma \\
z(0) & =0 & & \text { in } \Omega .
\end{aligned}\right.
$$

Proof. The proof is based on the implicit function theorem applied to the mapping

$$
\begin{aligned}
& \mathcal{F}: Y \times L^{2}\left(0, \infty ; L^{2}(\omega)\right) \longrightarrow L^{2}(Q) \times H^{1}(\Omega) \\
& \mathcal{F}(y, u)=\left(\frac{\partial y}{\partial t}-\Delta y+a y+f(y)-u \chi_{\omega}, y(0)-y_{0}\right) .
\end{aligned}
$$

The mapping $\mathcal{F}$ is well-defined. Indeed, the only delicate issue concerns the membership $f(y) \in L^{2}(Q)$. To prove this we use that $f(y)^{2} \leq C_{f}^{2}\left(y^{2}+y^{4 m+2}\right)$, as established in the proof of Theorem 2.4. Thus, it suffices to verify that $y^{4 m+2} \in L^{1}(Q)$. Recalling that $4 m+2 \leq 6$ for $n=3$, this is obtained as follows:

$$
\int_{Q} y^{4 m+2} d x d t \leq C \int_{0}^{\infty}\|y(t)\|_{H^{1}(\Omega)}^{4 m+2} d t \leq C\|y\|_{L^{\infty}\left(0, \infty ; H^{1}(\Omega)\right)}^{4 m}\|y\|_{L^{2}\left(0, \infty ; H^{1}(\Omega)\right)}^{2}<\infty .
$$

The above argument, in particular, implies that $Y$ is continuously embedded in $L^{4 m+2}(Q)$. It is easy to check that the mapping $y \in L^{4 m+2}(Q) \cap L^{2}(Q) \rightarrow f(y) \in$ $L^{2}(Q)$ is of class $C^{1}$. Hence, we have that $\mathcal{F}$ is also of class $C^{1}$, and the first partial derivative of $\mathcal{F}$ with respect to $y$ at $(y, u)$ in a direction $z \in Y$ is given by

$$
\frac{\partial \mathcal{F}}{\partial y}(y, u) z=\left(\frac{\partial z}{\partial t}-\Delta z+a z+f^{\prime}(y) z, z(0)\right) .
$$

Now for any $u \in \mathcal{U}$ we have that $\mathcal{F}\left(y_{u}, u\right)=\mathcal{F}(G(u), u)=(0,0)$. To complete the verification of the assumptions of the implicit function theorem it remains to prove that $\frac{\partial \mathcal{F}}{\partial y}\left(y_{u}, u\right): Y \longrightarrow L^{2}(Q) \times H^{1}(\Omega)$ is an isomorphism or, equivalently, that the equation

$$
\left\{\begin{aligned}
\frac{\partial z}{\partial t}-\Delta z+a z+f^{\prime}\left(y_{u}\right) z & =g & & \text { in } Q, \\
\partial_{n} z & =0 & & \text { on } \Sigma, \\
z(0) & =z_{0} & & \text { in } \Omega,
\end{aligned}\right.
$$

has a unique solution in $Y$ for every $\left(g, z_{0}\right) \in L^{2}(Q) \times H^{1}(\Omega)$. This is done in Lemma 3.5 below.

Lemma 3.5. For every $\left(g, z_{0}\right) \in L^{2}(Q) \times H^{1}(\Omega)(3.2)$ has a unique solution $z \in Y$. Moreover, there exists a constant independent of $\left(g, z_{0}\right)$ such that

$$
\|z\|_{Y} \leq C\left(\|g\|_{L^{2}(Q)}+\left\|z_{0}\right\|_{H^{1}(\Omega)}\right) .
$$

Proof. From our assumptions on $f$ we infer the existence of a polynomial $p$ of degree $2 m-2$ such that

$$
f^{\prime}(s)=(2 m+1) a_{2 m+1} s^{2 m}+a_{1}+p(s) s \geq p(s) s \text { because } a_{2 m+1}>0 \text { and } a_{1}>0 .
$$


Moreover there exists a constant $C_{f}^{\prime}>0$ such that $\left|f^{\prime}(s)\right| \leq C_{f}^{\prime}\left(s^{2 m}+1\right)$. We observe that $m=1$ in dimension $n=3$ and consequently $p$ is a constant in that case.

Since $y_{u} \in Y$, given $\varepsilon>0$ we can use (2.8) to deduce the existence of $T_{\varepsilon}>0$ so that

$$
\left\|y_{u}(t)\right\|_{L^{2}(\Omega)}<\varepsilon \quad \forall t \geq T_{\varepsilon} .
$$

Let us take $T>T_{\varepsilon}$ arbitrary. From (2.9) we have that $y_{u}^{2 m+1} \in L^{2}(Q)$, hence, $f^{\prime}\left(y_{u}\right) \in L^{2+\frac{1}{m}}\left(Q_{T}\right)$. From the classical theory for linear parabolic equations (see, for instance, [11, Chapter III]) we deduce the existence of a unique solution $z \in W(0, T)$ of (3.2) with

$$
W(0, T)=\left\{z \in L^{2}\left(0, T ; H^{1}(\Omega)\right): \frac{\partial z}{\partial t} \in L^{2}\left(0, T ; H^{1}(\Omega)^{*}\right)\right\} .
$$

Moreover we have that

$$
\|z\|_{W(0, T)} \leq C_{T}\left(\|g\|_{L^{2}\left(Q_{T}\right)}+\left\|z_{0}\right\|_{L^{2}(\Omega)}\right) .
$$

To obtain the estimates for $z$ in $Q$ we introduce for every $t>0$ the following sets,

$$
\Omega_{t}=\left\{x \in \Omega:\left|y_{u}(x, t)\right|<M_{0}\right\},
$$

where $M_{0}$ was given in (2.12). Set $C_{p}=\max _{|s| \leq M_{0}}|p(s)|$. Now, multiplying (3.2) by $z$, integrating by parts in $Q_{T}$, and using that $f^{\prime}\left(y_{u}\right) z^{2} \geq p\left(y_{u}\right) y_{u} z^{2}$ in $Q$ and $f^{\prime}\left(y_{u}(x, t)\right) \geq 0$ for every $x \in \Omega \backslash \Omega_{t}$ we get

$$
\begin{aligned}
& \frac{1}{2}\|z(T)\|_{L^{2}(\Omega)}^{2}+C_{a}\|z\|_{L^{2}\left(0, T ; H^{1}(\Omega)\right)}^{2} \\
& \leq \frac{1}{2}\left\|z_{0}\right\|_{L^{2}(\Omega)}^{2}+\int_{Q_{T}} g z d x d t-\int_{0}^{T} \int_{\Omega_{t}} p\left(y_{u}\right) y_{u} z^{2} d x d t \\
& \leq \frac{1}{2}\left\|z_{0}\right\|_{L^{2}(\Omega)}^{2}+\|g\|_{L^{2}\left(Q_{T}\right)}\|z\|_{L^{2}\left(Q_{T}\right)} \\
&+\int_{0}^{T_{\varepsilon}} \int_{\Omega_{t}}\left|p\left(y_{u}\right) y_{u}\right| z^{2} d x d t+\int_{T_{\varepsilon}}^{T} \int_{\Omega_{t}}\left|p\left(y_{u}\right) y_{u}\right| z^{2} d x d t \\
& \leq \frac{1}{2}\left\|z_{0}\right\|_{L^{2}(\Omega)}^{2}+\|g\|_{L^{2}\left(Q_{T}\right)}\|z\|_{L^{2}\left(0, T ; H^{1}(\Omega)\right)} \\
&+C_{p} M_{0}\|z\|_{L^{2}\left(Q_{T_{\varepsilon}}\right)}^{2}+C_{p} \int_{T_{\varepsilon}}^{T}\left\|y_{u}(t)\right\|_{L^{2}(\Omega)}\|z(t)\|_{L^{4}(\Omega)}^{2} d t \\
& \leq \frac{1}{2}\left\|z_{0}\right\|_{L^{2}(\Omega)}^{2}+\frac{1}{C_{a}}\|g\|_{L^{2}(Q)}^{2}+\frac{C_{a}}{4}\|z\|_{L^{2}\left(0, T ; H^{1}(\Omega)\right)}^{2} \\
&+C_{p} M_{0}\|z\|_{L^{2}\left(Q_{T_{\varepsilon}}\right)}^{2}+C_{p} \varepsilon\|z\|_{L^{2}\left(0, T ; H^{1}(\Omega)\right)}^{2},
\end{aligned}
$$

where $C_{a}$ is the constant that appeared in (2.4). Taking $\varepsilon=\frac{C_{a}}{4 C_{p}}$ we infer from the above inequality and (3.5) that

$$
\begin{aligned}
\|z\|_{L^{2}\left(0, T ; H^{1}(\Omega)\right)} & \leq \frac{1}{\sqrt{C_{a}}}\left(\left\|z_{0}\right\|_{L^{2}(\Omega)}+\frac{\sqrt{2}}{\sqrt{C_{a}}}\|g\|_{L^{2}(Q)}+\sqrt{2 C_{p} M_{0}}\|z\|_{L^{2}\left(Q_{T_{\varepsilon}}\right)}\right) \\
& \leq C^{\prime}\left(\|g\|_{L^{2}(Q)}+\left\|z_{0}\right\|_{L^{2}(\Omega)}\right) \quad \forall T>T_{\varepsilon} .
\end{aligned}
$$


Hence $z \in L^{2}\left(0, \infty ; H^{1}(\Omega)\right)$ holds. Next we prove that $z \in Y$. To this end we first establish that $f^{\prime}\left(y_{u}\right) z \in L^{2}(Q)$. Since

$$
f^{\prime}\left(y_{u}\right)^{2} z^{2} \leq{C_{f}^{\prime}}^{2}\left(y_{u}^{2 m}+1\right)^{2} z^{2} \leq 2{C_{f}^{\prime}}^{2}\left(y_{u}^{4 m}+1\right) z^{2}
$$

it is enough to prove that $y^{4 m} z^{2} \in L^{1}(Q)$. Using the Hölder inequality with $p=\frac{4 m+2}{4 m}$ and $p^{\prime}=2 m+1$, this is obtained as follows:

$$
\begin{aligned}
\int_{Q} y_{u}^{4 m} z^{2} d x d t & \leq \int_{0}^{\infty}\left\|y_{u}(t)\right\|_{L^{4 m+2}(\Omega)}^{4 m}\|z(t)\|_{L^{4 m+2}(\Omega)}^{2 m} d t \\
& \leq C \int_{0}^{\infty}\left\|y_{u}(t)\right\|_{H^{1}(\Omega)}^{4 m}\|z(t)\|_{H^{1}(\Omega)}^{2} d t \\
& \leq C\left\|y_{u}\right\|_{L^{\infty}\left(0, \infty ; H^{1}(\Omega)\right)}^{4 m}\|z\|_{L^{2}\left(0, \infty ; H^{1}(\Omega)\right)}^{2} .
\end{aligned}
$$

Additionally, with (3.6) we get the estimate

$$
\left\|f^{\prime}\left(y_{u}\right) z\right\|_{L^{2}(Q)} \leq C^{\prime \prime}\left(\left\|y_{u}\right\|_{L^{\infty}\left(0, \infty ; H^{1}(\Omega)\right)}^{2 m}+1\right)\left(\|g\|_{L^{2}(Q)}+\left\|z_{0}\right\|_{L^{2}(\Omega)}\right) .
$$

Finally, the regularity $\frac{\partial z}{\partial t} \in L^{2}(Q)$ and $z \in L^{\infty}\left(0, \infty ; H^{1}(\Omega)\right)$ and the corresponding estimates are proved as in the last two steps of the proof of Theorem 2.4, just taking into account the obtained a priori estimates (3.6) and (3.7).

Remark 3.6. Let us note that the assumption $m=1$ in the case $n=3$ was crucial in the proof of Theorem 3.4 to deduce that $\|y\|_{L^{4 m+2}(Q)}<\infty$.

3.3. Adjoint state equation. Let $u \in \mathcal{U}$ and $y_{u}$ be the associated state. We denote by $\varphi_{u}$ the adjoint state to $y_{u}$, which is the solution in $Y$ to

$$
\left\{\begin{aligned}
-\frac{\partial \varphi}{\partial t}-\Delta \varphi+a \varphi+f^{\prime}\left(y_{u}\right) \varphi & =y_{u} & & \text { in } Q, \\
\partial_{n} \varphi & =0 & & \text { on } \Sigma .
\end{aligned}\right.
$$

We observe that for any element $\varphi \in Y$ we have that $\frac{\partial \varphi}{\partial t}, \Delta \varphi \in L^{2}(Q)$, hence, the first equation in (3.8) is interpreted in $L^{2}(Q)$, and the second in the $L^{2}\left(0, \infty ; H^{-\frac{1}{2}}(\Gamma)\right)$ sense. Moreover, since $\varphi \in Y$ we recall that $\lim _{t \rightarrow \infty}\|\varphi(t)\|_{L^{2}(\Omega)}=0$.

Theorem 3.7. Equation (3.8) has a unique solution $\varphi_{u} \in Y$ and

$$
\left\|\varphi_{u}\right\|_{Y} \leq C\left\|y_{u}\right\|_{L^{2}(Q)}
$$

for some constant independent of $u \in \mathcal{U}$. Moreover the following identity holds:

$$
\int_{Q} \varphi_{u}\left(\frac{\partial z}{\partial t}-\Delta z+a z+f^{\prime}\left(y_{u}\right) z\right) d x d t=\int_{Q} y_{u} z d x d t \quad \forall z \in Y_{0}
$$

where $Y_{0}=\{z \in Y: z(0)=0$ in $\Omega\}$.

Proof. For every $T>0$ we consider the auxiliary equation

$$
\left\{\begin{aligned}
\frac{\partial z_{T}}{\partial t}-\Delta z_{T}+a z_{T}+f^{\prime}\left(y_{T}\right) z_{T} & =y_{T} & & \text { in } Q_{T} \\
\partial_{n} z_{T} & =0 & & \text { on } \Sigma_{T}, \\
z_{T}(0) & =0 & & \text { in } \Omega,
\end{aligned}\right.
$$


where $y_{T}(x, t)=y_{u}(x, T-t) \forall(x, t) \in Q_{T}$. As in Lemma 3.5 we have that this equation has a unique solution $z_{T} \in W(0, T)$ and the estimates (3.5)-(3.7) become in this case for every $T$

$$
\left\|z_{T}\right\|_{W(0, T)}+\left\|f^{\prime}\left(y_{T}\right) z_{T}\right\|_{L^{2}\left(Q_{T}\right)} \leq C\left\|y_{T}\right\|_{L^{2}\left(Q_{T}\right)} \leq C\left\|y_{u}\right\|_{L^{2}(Q)} .
$$

From these estimates and arguing as in the proof of Theorem 2.4 we get

$$
\left\|z_{T}\right\|_{L^{\infty}\left(0, T ; H^{1}(\Omega)\right)}+\left\|\frac{\partial z_{T}}{\partial t}\right\|_{L^{2}\left(Q_{T}\right)} \leq C^{\prime}\left\|y_{u}\right\|_{L^{2}(Q)}
$$

Now we take

$$
\varphi_{T}(x, t)=\left\{\begin{array}{cl}
z_{T}(x, T-t) & \text { if } t \in[0, T] \\
0 & \text { if } t>T
\end{array}\right.
$$

From the above estimates for $z_{T}$ we deduce that $\varphi_{T} \in Y$ and $\left\|\varphi_{T}\right\|_{Y} \leq C^{\prime \prime}\left\|y_{u}\right\|_{L^{2}(Q)}$. Moreover $\varphi_{T}$ satisfies

$$
\left\{\begin{aligned}
-\frac{\partial \varphi_{T}}{\partial t}-\Delta \varphi_{T}+a \varphi_{T}+f^{\prime}\left(y_{u}\right) \varphi_{T} & =y_{u} & & \text { in } Q_{T} \\
\partial_{n} \varphi_{T} & =0 & & \text { on } \Sigma_{T} \\
\varphi_{T}(T) & =0 & & \text { in } \Omega .
\end{aligned}\right.
$$

Now we take a sequence $T_{k} \rightarrow \infty$ such that $\varphi_{T_{k}} \rightarrow \varphi$ in $Y$. It is immediate to pass to the limit in the equations satisfied by the functions $\varphi_{T_{k}}$ and to deduce that $\varphi$ is a solution to (3.8) and (3.9) is satisfied. To verify (3.10) let us note that

$$
\int_{Q}\left(\frac{\partial \varphi_{T}}{\partial t}-\Delta \varphi_{T}+a \varphi_{T}+f^{\prime}\left(y_{u}\right) \varphi_{T}\right) z d x d t=\int_{Q} y_{u} z d x d t \quad \forall z \in Y_{0}
$$

which implies that

$$
\int_{0}^{T} \int_{\Omega} \varphi_{T}\left(\frac{\partial z}{\partial t}-\Delta z+a z+f^{\prime}\left(y_{u}\right) z\right) d x d t=\int_{0}^{T} \int_{\Omega} y_{u} z d x d t \quad \forall z \in Y_{0}
$$

Passing to the limit $T \rightarrow \infty$ we obtain (3.10). Finally if $\varphi_{u}^{1}$ and $\varphi_{u}^{2}$ are two solutions of the adjoint equation then

$$
\int_{Q}\left(\varphi_{u}^{1}-\varphi_{u}^{2}\right)\left(\frac{\partial z}{\partial t}-\Delta z+a z+f^{\prime}\left(y_{u}\right) z\right) d x d t=0 \quad \forall z \in Y_{0}
$$

Since the mapping of $z \rightarrow \frac{\partial z}{\partial t}-\Delta z+a z+f^{\prime}\left(y_{u}\right) z$ from $Y_{0}$ to $L^{2}(Q)$ is surjective by Lemma 3.5, we obtain that $\varphi_{u}^{1}=\varphi_{u}^{2}$. This concludes the proof.

4. Optimality conditions and sparsity. Before establishing the optimality conditions we analyze the cost functional $J$. We distinguish two terms in the functional: $J(u)=F(u)+\alpha j(u)$ with

$$
\begin{aligned}
& F(u)=\frac{1}{2} \int_{0}^{\infty} \int_{\Omega} y_{u}^{2} d x d t+\frac{\nu}{2} \int_{0}^{\infty} \int_{\omega} u^{2} d x d t \text { and } \\
& j(u)=\int_{0}^{\infty}\left(\int_{\omega} u^{2} d x\right)^{1 / 2} d t=\int_{0}^{\infty}\|u(t)\|_{L^{2}(\omega)} d t .
\end{aligned}
$$


Proposition 4.1. The function $F: \mathcal{U} \longrightarrow \mathbb{R}$ is of class $C^{1}$ and

$$
F^{\prime}(u) v=\int_{0}^{\infty} \int_{\omega}\left(\varphi_{u}+\nu u\right) v d x d t \quad \forall u \in \mathcal{U} \text { and } \forall v \in L^{2}\left(0, \infty, L^{2}(\omega)\right) .
$$

Proof. With the notation of Theorem 3.4 we have that

$$
F(u)=\frac{1}{2}\|G(u)\|_{L^{2}(Q)}^{2}+\frac{\nu}{2}\|u\|_{L^{2}\left(0, \infty ; L^{2}(\omega)\right)}^{2} .
$$

Hence, we deduce from the chain rule and Theorem 3.4 that $F$ is of class $C^{1}$ and

$$
F^{\prime}(u) v=\int_{Q} y_{u} z_{v} d x d t+\nu \int_{0}^{\infty} \int_{\omega} u v d x d t .
$$

Then, taking $\varphi_{u} \in Y$ as the solution of (3.8), noting that $z_{v} \in Y_{0}$, and using (3.1) and (3.10) we obtain (4.1).

Now we study the functional $j: L^{1}\left(0, \infty ; L^{2}(\Omega)\right) \longrightarrow \mathbb{R}$. This functional is not differentiable at every point of the domain, but it is convex and Lipschitz. Therefore there exist the directional derivatives $j^{\prime}(u ; v)$ for every $u, v \in L^{1}\left(0, \infty ; L^{2}(\Omega)\right)$ and the subdifferential $\partial j(u)$ is nonempty for every $u$. Let us characterize these objects.

Given an element $u \in L^{1}\left(0, \infty ; L^{2}(\omega)\right)$, we denote

$$
I_{u}=\left\{t \in(0, \infty):\|u(t)\|_{L^{2}(\omega)} \neq 0\right\} \text { and } I_{u}^{0}=(0, \infty) \backslash I_{u} .
$$

Proposition 4.2. The following statements hold.

1. $\lambda \in \partial j(u)$ is equivalent to $\lambda \in L^{\infty}\left(0, \infty ; L^{2}(\omega)\right)$ and

$$
\begin{cases}\|\lambda(t)\|_{L^{2}(\omega)} \leq 1 & \text { for a.a. } t \in I_{u}^{0}, \\ \lambda(x, t)=\frac{u(x, t)}{\|u(t)\|_{L^{2}(\omega)}} \quad \text { for a.a. }(x, t) \in \omega \times I_{u} .\end{cases}
$$

2. For every $u, v \in L^{1}\left(0, \infty ; L^{2}(\omega)\right)$

$$
j^{\prime}(u ; v)=\int_{I_{u}^{0}}\|v(t)\|_{L^{2}(\omega)} d t+\int_{I_{u}} \frac{1}{\|u(t)\|_{L^{2}(\omega)}}\left(\int_{\omega} u v d x\right) d t .
$$

The reader is referred to [6, Proposition 2.8] for the proof of this result where the role of $x$ and $t$ are reversed.

Now we are prepared to establish the optimality conditions for a local solution of (P) in the sense of $L^{2}\left(0, \infty ; L^{2}(\omega)\right)$.

TheOrem 4.3. Let $\bar{u}$ be a local solution of $(\mathrm{P})$. Then there exists $\bar{\lambda} \in \partial j(\bar{u})$ such that

$$
\bar{\varphi}+\nu \bar{u}+\alpha \bar{\lambda}=0 \text { in } \omega \times(0, \infty)
$$

where $\bar{\varphi}$ is the adjoint state associated with $\bar{y}=y_{\bar{u}}$.

Proof. For arbitrary $u \in U=L^{2}\left(0, \infty ; L^{2}(\omega)\right) \cap L^{1}\left(0, \infty ; L^{2}(\omega)\right)$ we have with (4.1) and the convexity of $j$

$$
\begin{aligned}
0 & \leq \lim _{\rho \searrow 0} \frac{J(\bar{u}+\rho(u-\bar{u}))-J(\bar{u})}{\rho} \\
& =\lim _{\rho \searrow 0} \frac{F(\bar{u}+\rho(u-\bar{u}))-F(\bar{u})}{\rho}+\alpha \lim _{\rho \searrow 0} \frac{j(\bar{u}+\rho(u-\bar{u}))-j(\bar{u})}{\rho} \\
& \leq F^{\prime}(\bar{u})(u-\bar{u})+\alpha[j(u)-j(\bar{u})]=\int_{0}^{\infty} \int_{\omega}(\bar{\varphi}+\nu \bar{u})(u-\bar{u}) d x d t+\alpha[j(u)-j(\bar{u})] .
\end{aligned}
$$


We set $\bar{\lambda}=-\frac{1}{\alpha}(\bar{\varphi}+\nu \bar{u}) \chi_{\omega} \in L^{2}\left(0, \infty ; L^{2}(\omega)\right)$. Then the above inequality implies that

$$
\int_{0}^{\infty} \int_{\omega} \bar{\lambda}(u-\bar{u}) d x d t+j(\bar{u}) \leq j(u) \quad \forall u \in U .
$$

Let us check that $\bar{\lambda} \in \partial j(\bar{u})$. To this end we need to prove that $\bar{\lambda} \in L^{\infty}\left(0, \infty ; L^{2}(\omega)\right)$. We define

$$
E=\left\{t \in(0, \infty):\|\bar{\lambda}(t)\|_{L^{2}(\omega)}>1\right\} .
$$

We will prove that $|E|=0$. Since $\bar{\lambda} \in L^{2}\left(0, \infty ; L^{2}(\omega)\right)$ we have that $|E|<\infty$. Set

$$
v(x, t)=\left\{\begin{array}{cl}
\bar{\lambda}(x, t) & \text { if } t \in E, \\
0 & \text { otherwise, }
\end{array} \quad \text { and } u=\bar{u}+v \in U .\right.
$$

Putting this $u$ in (4.5) we get

$$
\begin{aligned}
\int_{E}\|\bar{\lambda}(t)\|_{L^{2}(\omega)}^{2} d t & =\int_{0}^{\infty} \int_{\omega} \bar{\lambda} v d x d t \\
& =\int_{0}^{\infty} \int_{\omega} \bar{\lambda}(u-\bar{u}) d x d t \leq j(u)-j(\bar{u}) \leq j(v) \\
& =\int_{0}^{\infty}\|v(t)\|_{L^{2}(\omega)} d t=\int_{E}\|\bar{\lambda}(t)\|_{L^{2}(\omega)} d t
\end{aligned}
$$

which is a contradiction to the definition of $E$ unless $|E|=0$. Thus $\|\bar{\lambda}\|_{L^{\infty}\left(0, \infty ; L^{2}(\omega)\right)} \leq$ 1 holds. Finally, it is enough to use the density of $U$ in $L^{1}\left(0, \infty ; L^{2}(\omega)\right)$ to deduce that (4.5) holds $\forall u \in L^{1}\left(0, \infty ; L^{2}(\omega)\right)$, and hence $\bar{\lambda} \in \partial j(\bar{u})$.

Corollary 4.4. Let $(\bar{u}, \bar{\varphi}, \bar{\lambda})$ be as in Theorem 4.3. Then the following properties hold:

$$
\begin{aligned}
\|\bar{u}(t)\|_{L^{2}(\omega)} & =0 \Leftrightarrow\|\bar{\varphi}(t)\|_{L^{2}(\omega)} \leq \alpha, \\
\bar{\lambda}(x, t) & =\left\{\begin{array}{c}
-\frac{1}{\alpha} \bar{\varphi}(x, t) \quad \text { if } t \in I_{\bar{u}}^{0}, \\
\frac{\bar{u}(x, t)}{\|\bar{u}(t)\|_{L^{2}(\omega)}} \quad \text { if } t \in I_{\bar{u}},
\end{array}\right. \\
\bar{u}(x, t) & =-\frac{\|\bar{u}(t)\|_{L^{2}(\omega)} \bar{\varphi}(x, t)}{\nu\|\bar{u}(t)\|_{L^{2}(\omega)}+\alpha}
\end{aligned}
$$

for almost every $x \in \omega$. Moreover $\bar{\lambda}, \bar{u} \in C\left([0, \infty) ; L^{2}(\omega)\right)$ and

$$
\|\bar{u}\|_{L^{\infty}\left(0, \infty ; L^{2}(\omega)\right)} \leq \frac{1}{\nu}\|\bar{\varphi}\|_{L^{\infty}\left(0, \infty ; L^{2}(\omega)\right)} .
$$

Proof. First we observe that (4.7) is an immediate consequence of (4.2) and (4.4). Combining (4.4) and (4.7) we infer

$$
\bar{\varphi}(x, t)+\bar{u}(x, t)\left[\nu+\frac{\alpha}{\|\bar{u}(t)\|_{L^{2}(\omega)}}\right]=0 \text { for a.a. }(x, t) \in \omega \times I_{\bar{u}} .
$$

Taking norms in this identity we get

$$
\|\bar{\varphi}(t)\|_{L^{2}(\omega)}=\nu\|\bar{u}(t)\|_{L^{2}(\omega)}+\alpha \text { for a.a. } t \in I_{\bar{u}} .
$$


From (4.7) and (4.2) we have that

$$
\|\bar{\varphi}(t)\|_{L^{2}(\omega)}=\alpha\|\bar{\lambda}(t)\|_{L^{2}(\omega)} \leq \alpha \quad \text { for a.a. } t \in I_{\bar{u}}^{0} .
$$

Since $\bar{u}$ is zero in $I_{\bar{u}}^{0}$ we get from the above relationship and (4.11)

$$
\|\bar{u}(t)\|_{L^{2}(\Omega)}=\frac{1}{\nu} \max \left\{0,\|\bar{\varphi}(t)\|_{L^{2}(\omega)}-\alpha\right\} \text { for a.a. } t \in(0, \infty) .
$$

From here (4.6) follows. Moreover, since $\bar{\varphi} \in C\left(\left[0, \infty ; L^{2}(\Omega)\right)\right.$ we deduce that the function $t \in[0, \infty) \rightarrow\|\bar{u}(t)\|_{L^{2}(\omega)} \in \mathbb{R}$ is continuous. Now, from (4.10) we obtain that (4.8) holds in $I_{\bar{u}}$. But, taking into account that $\bar{u}$ is zero in $I_{\bar{u}}^{0}$, we conclude that the identity (4.8) holds a.e. in $[0, \infty)$. Additionally, the continuity of $t \in[0, \infty) \rightarrow\|\bar{u}(t)\|_{L^{2}(\omega)} \in \mathbb{R}$ and the property $\bar{\varphi} \in C\left([0, \infty) ; L^{2}(\Omega)\right)$ imply that $\bar{u} \in C\left([0, \infty) ; L^{2}(\omega)\right)$. From (4.4) the same regularity follows for $\bar{\lambda}$. Finally, (4.9) is an immediate result of (4.8).

Remark 4.5. Let us observe that $\bar{\varphi} \in Y$ and consequently

$$
\lim _{t \rightarrow \infty}\|\bar{\varphi}(t)\|_{L^{2}(\omega)} \leq \lim _{t \rightarrow \infty}\|\bar{\varphi}(t)\|_{L^{2}(\Omega)}=0 .
$$

Hence, there exists $T_{\alpha}>0$ such that $\|\bar{\varphi}(t)\|_{L^{2}(\omega)} \leq \alpha$ for all $t \geq T_{\alpha}$. Then (4.6) implies that $\bar{u}(x, t)=0$ at least for all $t \geq T_{\alpha}$. This proves the sparsity of the optimal control.

\section{REFERENCES}

[1] V. Barbu and G. WANG, Internal stabilization of semilinear parabolic systems, J. Math. Anal. Appl., 285 (2003), pp. 387-407.

[2] V. Barbu And G. Wang, Feedback stabilization of semilinear heat equations, Abstr. Appl. Anal., (2003), pp. 697-714.

[3] A. J. V. Brandão, E. Fernández-Cara, P. M. D. Magalhães, and M. A. Rojas-Medar, Theoretical analysis and control results for the FitzHugh-Nagumo equation, Electron. J. Differential Equations, 2008 (2008), 164, pp. 1-20.

[4] H. Brezis, Functional Analysis, Sobolev Spaces and Partial Differential Equations, Springer, New York, 2011.

[5] E. Casas, C. Clason, And K. Kunisch, Parabolic control problems in measure spaces with sparse solutions, SIAM J. Control Optim., 51 (2013), pp. 28-63.

[6] E. Casas, R. Herzog, and G. Wachsmuth, Analysis of spatio-temporally sparse optimal control problems of semilinear parabolic equations, ESAIM Control Optim. Calc. Var., 23 (2017), pp. 263-295.

[7] E. Casas And K. Kunisch, Parabolic control problems in space-time measure spaces, ESAIM Control Optim. Calc. Var., 22 (2016). pp. 355-370.

[8] E. Casas, C. Ryll, and F. Tröltzsch, Second order and stability analysis for optimal sparse control of the FitzHugh-Nagumo equation, SIAM J. Control Optim., 53 (2015), pp. 21682202.

[9] R. Herzog, G. Stadler, and G. Wachsmuth, Directional sparsity in optimal control of partial differential equations, SIAM J. Control Optim., 50 (2012), pp. 943-963.

[10] K. Kunisch, K. Pieper, And B. Vexler, Measure valued directional sparsity for parabolic optimal control problems, SIAM J. Control Optim., 52 (2014), pp. 3078-3108.

[11] O. Ladyzhenskaya, V. Solonnikov, and N. Ural'tseva, Linear and Quasilinear Equations of Parabolic Type, AMS, Providence, RI, 1988.

[12] J. D. Murray, Mathematical Biology. I. An Introduction, Interdisciplinary Appl. Math. 17, Springer, New York, 2002.

[13] C. Nagaiah, K. Kunisch, and G. Plank, Optimal control approach to termination of re-entry waves in cardiac electrophysiology, J. Math. Biol., 67 (2013), pp. 359-388.

[14] F. SCHLÖGL, A characteristic critical quantity in nonequilibrium phase transitions, Z. Phys. B Condens. Matter, 52 (2004), pp. 51-60.

[15] R. E. Showalter, Monotone Operators in Banach Space and Nonlinear Partial Differential Equations, Math. Surv. Monogr. 49, AMS, Providence, RI, 1997.

[16] M. SîrBu, Infinite time horizon optimal control of the semilinear heat equation, Nonlinear Funct. Anal. Appl., 7 (2002), pp. 69-83. 